\title{
Weak and strong convergence theorems for nonlinear mappings and system of generalized mixed equilibrium problems in Hilbert spaces
}

\author{
Qingqing Cheng ${ }^{a, *}$, Yongfu Su \\ a Department of Mathematics and LPMC, Nankai University, Tianjin, 300071, China. \\ ${ }^{b}$ Department of Mathematics, Tianjin Polytechnic University, Tianjin, 300387, China. \\ Communicated by X. Qin
}

\begin{abstract}
In this paper, we construct two iteration schemes for approximating a common element of the set of solutions of equilibrium problems (GMEP and GEP) and the set of common fixed points of a finite family of k-strictly asymptotically pseudo-contractions in Hilbert spaces. Fixed point theorems are established in Hilbert spaces. Numerical examples and applications are provided. The main results of this paper modify and improve many important recent results in the literature. (C) 2017 All rights reserved.
\end{abstract}

Keywords: Equilibrium problem, Modified Ishikawa's iteration, hybrid algorithm, Hilbert space, weak and strong convergence.

2010 MSC: 47H05, 47H09, 47H10.

\section{Introduction}

Let $\mathrm{H}$ be an infinite dimensional real Hilbert space. Let $\mathrm{C}$ be a nonempty subset of $\mathrm{H}$ and let $\mathrm{T}: \mathrm{C} \rightarrow \mathrm{C}$ be a mapping. Recall that $\mathrm{T}$ is said to be nonexpansive if and only if

$$
\|\mathrm{T} x-\mathrm{T} y\| \leqslant\|x-y\|, \quad \forall x, y \in C, \quad \forall n \geqslant 1 .
$$

$\mathrm{T}$ is said to be asymptotically nonexpansive if and only if there exists a sequence $\left\{k_{n}\right\} \subset[1, \infty)$ such that

$$
\left\|T^{n} x-T^{n} y\right\| \leqslant k_{n}\|x-y\|, \quad \forall x, y \in C, \quad \forall n \geqslant 1 .
$$

A mapping $T$ is called a k-strictly pseudocontractive mapping if and only if there exists a constant $k \in$ $[0,1)$ such that

$$
\|T x-T y\|^{2} \leqslant\|x-y\|^{2}+k\|x-y-(T x-T y)\|^{2}, \quad \forall x, y \in C .
$$

A mapping $T$ is called a $k$-strictly asymptotically pseudocontractive mapping if and only if there exist a constant $k \in[0,1)$ and a sequence $\left\{k_{n}\right\} \subseteq[1, \infty)$ with $\lim _{n \rightarrow \infty} k_{n}=1$ such that

$$
\left\|T^{n} x-T^{n} y\right\|^{2} \leqslant k_{n}^{2}\|x-y\|^{2}+k\left\|\left(I-T^{n}\right) x-\left(I-T^{n}\right) y\right\|^{2}, \quad \forall x, y \in C .
$$

\footnotetext{
*Corresponding author

Email addresses: chengqingqing2006@126.com (Qingqing Cheng), suyongfu@tjpu .edu.cn (Yongfu Su)
} doi:10.22436/jnsa.010.04.13 
Recall that $\mathrm{T}$ is called uniformly L-Lipschitzian if and only if there exists some $\mathrm{L}>0$ such that

$$
\left\|T^{n} x-T^{n} y\right\| \leqslant L\|x-y\|, \quad \forall x, y \in C, \quad \forall n \geqslant 1 .
$$

It is obvious to observe that

(i) $\mathrm{T}$ is a k-strictly pseudocontractive mapping when $\mathrm{k}_{\mathrm{n}} \equiv 1$;

(ii) $\mathrm{T}$ is an asymptotically nonexpansive mapping when $\mathrm{k}=0$;

(iii) $\mathrm{T}$ is a nonexpansive mapping when $k_{\mathrm{n}} \equiv 1$ and $k=0$;

(iv) if $\left\{T_{i}\right\}_{1 \leqslant i \leqslant N}$ is a finite family of $\left\{s_{i}\right\}_{1 \leqslant i \leqslant N}$-strictly asymptotically pseudo-contractive mappings with sequence $\left\{s_{i}\right\} \subseteq[0,1)$ and $\left\{k_{n, i}\right\} \subseteq[1, \infty)$ such that $\lim _{n \rightarrow \infty} k_{n, i}=1$, then we have

$$
\left\|T_{i}^{n} x-T_{i}^{n} y\right\|^{2} \leqslant k_{n}^{2}\|x-y\|^{2}+s\left\|\left(I-T_{i}^{n}\right) x-\left(I-T_{i}^{n}\right) y\right\|^{2}, \quad \forall x, y \in C,
$$

for all $1 \leqslant i \leqslant N$, where constant $s=\max \left\{s_{i}: 1 \leqslant i \leqslant N\right\}$ and sequence $\left\{k_{n}\right\}=\max \left\{k_{n, i}: 1 \leqslant i \leqslant N\right\}$ with $\lim _{n \rightarrow \infty} k_{n}=1$.

Recently, the class of k-strictly asymptotically pseudocontractive mappings has been extensively investigated by many authors as an important extension of asymptotically nonexpansive mappings; see $[13,16,22,23]$ and the references therein. To study computational fixed points of nonlinear mappings, various iterative methods, such as mean valued iteration methods, projection iterative methods, splitting iterative methods, regularization iterative methods and so on, have been introduced and studied based on different analysis techniques; see $[5,7,12,18,21]$ and the references.

Recently, Qin et al. [16] constructed a new iterative algorithm for approximating common fixed points of a finite family of k-strictly asymptotically pseudocontractive mappings in real Hilbert spaces by generating the sequence $\left\{x_{n}\right\}$ as follows:

$$
x_{n}=\alpha_{n-1} x_{n-1}+\left(1-\alpha_{n-1}\right) T_{i(n)}^{h(n)} x_{n-1}, \quad n \geqslant 1 .
$$

Specifically,

$$
\left\{\begin{array}{l}
x_{1}=\alpha_{0} x_{0}+\left(1-\alpha_{0}\right) T_{1} x_{0} \\
x_{2}=\alpha_{1} x_{1}+\left(1-\alpha_{1}\right) T_{2} x_{1}, \\
\vdots \\
x_{N}=\alpha_{N-1} x_{N-1}+\left(1-\alpha_{N-1}\right) T_{N} x_{N-1}, \\
x_{N+1}=\alpha_{N} x_{N}+\left(1-\alpha_{N}\right) T_{1}^{2} x_{N}, \\
\vdots \\
x_{2 N}=\alpha_{2 N-1} x_{N-1}+\left(1-\alpha_{2 N-1}\right) T_{N}^{2} x_{2 N-1}, \\
x_{2 N+1}=\alpha_{2 N} x_{2 N}+\left(1-\alpha_{2 N}\right) T_{1}^{3} x_{2 N}, \\
\vdots
\end{array}\right.
$$

where we can write that $n=(h-1) N+i$, where $i=i(n) \in\{1,2, \ldots N\}, h=h(n) \geqslant 1$ is a positive integer and $h(n) \rightarrow \infty$ as $n \rightarrow \infty$. When sequence $\left\{\alpha_{n}\right\}$ satisfies certain conditions, the sequence $\left\{x_{n}\right\}$ generated by algorithm (1.1) converges weakly to a point in $\bigcap_{i=1}^{N} F\left(T_{i}\right)$.

Let $\mathrm{B}: \mathrm{C} \rightarrow \mathrm{H}$ be a monotone mapping, that is,

$$
\langle\mathrm{B} x-\mathrm{B} y, x-y\rangle \geqslant 0, \quad \forall x, y \in C .
$$

Let $\varphi: C \rightarrow R$ be a semicontinuous and convex functional and $\Theta: C \times C \rightarrow R$ be a real-valued bifunction. Assume that $\Theta$ satisfies the following conditions: 
(A1) $\Theta(x, x)=0, \forall x \in C$;

(A2) $\Theta$ is monotone, i.e., $\Theta(x, y)+\Theta(y, x) \leqslant 0, \forall x, y \in C$;

(A3) $\lim \sup _{t \rightarrow 0} \Theta(x+t(z-x), y) \leqslant \Theta(x, y), \forall x, y \in C$;

(A4) the function $y \mapsto \Theta(x, y)$ is convex and lower semicontinuous.

Recall the so-called the system of generalized mixed equilibrium problems (GMEP) is to find $x \in \mathrm{C}$ such that

$$
\Theta(x, y)+\langle B x, y-x\rangle+\varphi(y)-\varphi(x) \geqslant 0, \quad \forall y \in C .
$$

We use $\operatorname{GMEP}(\Theta, B, \varphi)$ to denote the set of solutions to (1.2), i.e.,

$$
\operatorname{GMEP}(\Theta, B, \varphi)=\{x \in C: \Theta(x, y)+\langle B x, y-x\rangle+\varphi(y)-\varphi(x) \geqslant 0, \forall y \in C\} .
$$

If $\varphi \equiv 0$, problem (1.2) turns into the mixed equilibrium problem for $\Theta$, B, denoted by $\operatorname{GEP}(\Theta, B)$ which is to find $x \in C$ such that

$$
\Theta(x, y)+\langle B x, y-x\rangle \geqslant 0, \quad \forall y \in C .
$$

If $\mathrm{B} \equiv 0$ and $\varphi \equiv 0$, problem (1.2) turns into the equilibrium problem for $\Theta$, denoted by $\operatorname{EP}(\Theta)$ which is to find $x \in C$ such that

$$
\Theta(x, y) \geqslant 0, \quad \forall y \in C .
$$

The generalized mixed equilibrium problem, which includes many important problems, for instance, complementarity problems, variational inequality problems, optimization problems, and fixed point problems as special cases, has been extensively investigated by many authors; see $[2,6,9,11]$ and the references therein. There are numerous problems in physics, optimization and economics which can be reduced to find a solution of generalized equilibrium problem. For exploring its solutions, various iterative methods have been proposed, see $[15,19,24]$ and the references therein.

Due to extensive applications of equilibrium problems and k-strictly asymptotically pseudo-contractions, the topic of approximating common element of the set of solutions of the equilibrium problem and the set of the fixed points of k-strictly asymptotically pseudo-contractions attract more attention recently. For solving these problems, Liu [13] proposed the following iterative method:

$$
\left\{\begin{array}{l}
x_{0} \in C \text { chosen arbitrarily, } \\
y_{n-1}=\alpha_{n-1} x_{n-1}+\left(1-\alpha_{n-1}\right) T_{i(n)}^{h(n)} x_{n-1}, \\
x_{n} \in C, \text { such that } \Theta\left(x_{n}, y\right)+\left\langle B y_{n-1}, y-x_{n}\right\rangle+\frac{1}{\lambda_{n-1}}\left\langle y-x_{n}, x_{n}-y_{n-1}\right\rangle \geqslant 0, \quad \forall y \in C,
\end{array}\right.
$$

where $\left\{T_{i}\right\}_{1 \leqslant i \leqslant N}$ is a finite family of strictly asymptotically pseudo-contractive mappings, $\varphi: C \rightarrow R$ is a proper lower semi-continuous and convex functional, $\mathrm{B}: \mathrm{C} \rightarrow \mathrm{H}$ is a continuous and monotone mapping and $\Theta: C \times C \rightarrow R$ satisfies (A1)-(A4). Under appropriate conditions imposed on sequences $\left\{\alpha_{n}\right\}$ and $\left\{\beta_{n}\right\}$ satisfied, they obtained weak and strong convergence theorems.

Motivated by the above mentioned results and the on-going research, we construct several new iteration schemes for approximating a common element of the set of solutions of equilibrium problems (GMEP and GEP) and the set of common fixed points of a finite family of k-strictly asymptotically pseudocontractions in Hilbert spaces.

The main contributions of this paper are the following:

(i) The Modified Mann iteration method in algorithm (1.3) is replaced by a new iteration in our paper. Moreover, we also consider approximating the common element of the set of solutions of generalized mixed equilibrium problems (GMEP), not only equilibrium problem (GEP), and the set of common fixed points of a finite family of k-strictly asymptotically pseudo-contractions. And we obtain two different weak convergence theorems. 
(ii) By means of hybrid algorithms, we obtain two strong convergence theorems corresponding to weak convergence theorems.

(iii) We apply the results to approximate the common element of the set of solutions of equilibrium problems (GMEP and GEP) and the set of common fixed points of a finite family of asymptotically nonexpansive mappings under suitable conditions.

(iv) We apply our results to (mixed) equilibrium problem (EP and MEP), (mixed) variational inequality, convex minimization problem and convex feasibility problem.

The rest of the paper is organized as follows: Section 2 describes several definitions and lemmas which we will use in proving our main results. Also, we give an example of a k-strictly asymptotically pseudocontractive mapping with nonempty set of fixed points to support our results. Section 3 presents our main results which include two weak convergence theorems and two strong convergence theorems. Section 4 introduces several interesting applications of our results. Finally, we conclude our paper in Section 5.

\section{Preliminaries}

Throughout the paper, $\rightarrow$ and $\rightarrow$ denote the strong convergence and weak convergence, respectively. In addition, $F(T)$ and $\omega_{w}\left(x_{n}\right)$ denote the fixed point set of $T$ and the weak $\omega$-limit set of $x_{n}$, respectively, that is, $F(T)=\{x \in C: T x=x\}$ and $\omega_{w}\left(x_{n}\right)=\left\{u: \exists x_{n_{j}} \rightarrow u\right\}$.

In an infinite dimensional real Hilbert space $H$, for all $x, y \in H$, the following properties hold:

$$
\begin{gathered}
\|x-y\|^{2}=\|x\|^{2}-\|y\|^{2}-2\langle x-y, y\rangle, \\
\|\lambda x+(1-\lambda) y\|^{2}=\lambda\|x\|^{2}+(1-\lambda)\|y\|^{2}-\lambda(1-\lambda)\|x-y\|^{2}, \quad \forall \lambda \in[0,1] .
\end{gathered}
$$

Let $\mathrm{C}$ be a nonempty closed and convex subset of $\mathrm{H}$, for each $x \in \mathrm{H}$, there exists a nearest point from $x$ to $C$. We denote the nearest point by $P_{C} x$, i.e., $\left\|x-P_{C} x\right\|=\inf \{\|x-y\|: y \in C\}$, where $P_{C}$ is called metric projection from $\mathrm{H}$ onto $\mathrm{C}$, and we have that

$$
\left\langle x-P_{C} x, P_{C} x-y\right\rangle \geqslant 0, \quad \forall y \in C .
$$

Let $\mathrm{B}$ be a mapping of $\mathrm{C}$ into $\mathrm{H}$. Recall that $\mathrm{B}$ is an $\alpha$-inverse-strongly monotone mapping if and only if there exists $\alpha>0$ such that

$$
\langle\mathrm{B} x-\mathrm{B} y, x-\mathrm{y}\rangle \geqslant \alpha\|\mathrm{B} x-\mathrm{B} y\|^{2}, \forall x, y \in \mathrm{C} .
$$

It is clear that if $B$ is an $\alpha$-inverse-strongly monotone mapping, then it must be a $\frac{1}{\alpha}$-Lipschitz operator. Moreover, for all $x, y \in C$ and $r>0$, we can observe that

$$
\begin{aligned}
\|(I-r B) x-(I-r B) y\|^{2} & =\|(x-y)-r(B x-B y)\|^{2} \\
& =\|x-y\|^{2}-2 r\langle x-y, B x-B y\rangle+r^{2}\|B x-B y\|^{2} \\
& \leqslant\|x-y\|^{2}+r(r-2 \alpha)\|B x-B y\|^{2} .
\end{aligned}
$$

From the last inequality, we can see that $\mathrm{I}-\mathrm{rB}: \mathrm{C} \rightarrow \mathrm{H}$ is nonexpansive when $r \leqslant 2 \alpha$. The class of inverse-strongly monotone mappings has recently extensively investigated by many authors in different framework of spaces; see $[3,8,17]$ and the references therein.

To obtain the main results of this paper, we also need the following lemmas:

Lemma 2.1 ([4, 10]). Let $\mathrm{C}$ be a nonempty closed convex subset of $\mathrm{H}$. Let $\Theta$ be a bifunction from $\mathrm{C} \times \mathrm{C} \rightarrow \mathrm{R}$ satisfies (A1)-(A4), and let $\varphi: \mathrm{C} \rightarrow \mathrm{R}$ be a proper lower semicontinuous and convex function. Let $\mathrm{B}: \mathrm{C} \rightarrow \mathrm{H}$ be a continuous monotone mapping. Then for $\mathrm{r}>0$ and $\mathrm{x} \in \mathrm{H}$, there exists $\mathrm{u} \in \mathrm{C}$ such that

$$
\Theta(\mathfrak{u}, \mathrm{y})+\langle\mathrm{Bu}, \mathrm{y}-\mathfrak{u}\rangle+\varphi(\mathrm{y})-\varphi(\mathfrak{u})+\frac{1}{\mathrm{r}}\langle\mathrm{y}-\mathrm{u}, \mathfrak{u}-\mathrm{x}\rangle \geqslant 0, \quad \forall \mathrm{y} \in \mathrm{C} .
$$


Define a mapping $\mathrm{K}_{\mathrm{r}}: \mathrm{H} \rightarrow \mathrm{C}$ as follows:

$$
K_{r} x:=\left\{u \in C: \Theta(u, y)+\langle B u, y-u\rangle+\varphi(y)-\varphi(u)+\frac{1}{r}\langle y-u, u-x\rangle \geqslant 0, \forall y \in C\right\}
$$

for all $\mathrm{x} \in \mathrm{H}$ and $\mathrm{r}>0$. Then, the following hold:

(i) For each $\mathrm{x} \in \mathrm{H}, \mathrm{K}_{\mathrm{r}}(\mathrm{x}) \neq \emptyset$;

(ii) $\mathrm{K}_{\mathrm{r}}$ is single-valued;

(iii) $\mathrm{K}_{\mathrm{r}}$ is firmly nonexpansive, that is, for any $\mathrm{x}, \mathrm{y} \in \mathrm{H}$,

$$
\left\|K_{r} x-K_{r} y\right\|^{2} \leqslant\left\langle K_{r} x-K_{r} y, x-y\right\rangle ;
$$

(iv) $\mathrm{F}\left(\mathrm{K}_{\mathrm{r}}\right)=\mathrm{GMEP}(\Theta, \varphi, \mathrm{B})$;

(v) $\operatorname{GMEP}(\Theta, \varphi, \mathrm{B})$ is closed and convex.

And notice that $\Omega(x, y)=\Theta(x, y)+\langle B x, y-x\rangle+\varphi(y)-\varphi(x)$ satisfies conditions (A1)-(A4) (see [10]). Additionally, if $\mathrm{B} \equiv 0$ and $\varphi \equiv 0, \operatorname{GMEP}(\Theta, \varphi, \mathrm{B})$ reduces to $\operatorname{EP}(\Theta)$, that is,

$$
\Theta(u, y)+\frac{1}{r}\langle y-u, u-x\rangle \geqslant 0, \quad \forall y \in C
$$

Then, define a mapping $\mathrm{T}_{\mathrm{r}}: \mathrm{H} \rightarrow \mathrm{C}$ and

$$
\mathrm{T}_{\mathrm{r}} \mathrm{x}:=\left\{\mathrm{u} \in \mathrm{C}: \Theta(\mathrm{u}, \mathrm{y})+\frac{1}{\mathrm{r}}\langle\mathrm{y}-\mathrm{u}, \mathrm{u}-\mathrm{x}\rangle \geqslant 0, \forall \mathrm{y} \in \mathrm{C}\right\}
$$

for all $x \in H$ and $r>0$. It is obvious that the above conclusions of Lemma 2.1 are also suitable for $\operatorname{EP}(\Theta)$.

Lemma 2.2 ([20]). Let $\left\{a_{n}\right\},\left\{b_{n}\right\}$ and $\left\{c_{n}\right\}$ are sequences of nonnegative real numbers satisfying the inequality

$$
a_{n+1} \leqslant\left(1+b_{n}\right) a_{n}+c_{n}, \quad \forall n \geqslant 0,
$$

if $\sum_{\mathfrak{n}=0}^{\infty} b_{\mathfrak{n}}<\infty$ and $\sum_{n=0}^{\infty} c_{n}<\infty$, then $\lim _{n \rightarrow \infty} a_{n}$ exists.

Lemma 2.3 ([16]). Let $\mathrm{C}$ be a closed convex subset of a real Hilbert space $\mathrm{H}$ and $\mathrm{T}$ be an asymptotically k-strictly pseudocontractive.

(i) T is uniformly L-Lipschitzian.

(ii) If $\mathrm{F}(\mathrm{T})$ is nonempty, then ( $\mathrm{I}-\mathrm{T})$ is demiclosed at zero, that is,

$$
x_{\mathrm{n}} \rightarrow \mathrm{u} \text { and }(\mathrm{I}-\mathrm{T}) \mathrm{x}_{\mathrm{n}} \rightarrow 0 \Rightarrow(\mathrm{I}-\mathrm{T}) \mathrm{u}=0 .
$$

(iii) $\mathrm{F}(\mathrm{T})$ is closed and convex so that the projection $\mathrm{P}_{\mathrm{F}(\mathrm{T})}$ is well-defined.

Lemma 2.4 ([11]). Let $\mathrm{C}$ be a closed convex subset of a real Hilbert space $\mathrm{H}$ and $\mathrm{x}, \mathrm{y}, z \in \mathrm{H}$. The set

$$
\left\{v \in C:\|y-v\|^{2} \leqslant\|x-v\|^{2}+\langle z, v\rangle+a\right\}
$$

is convex (and closed), where a is a real number.

Lemma 2.5 ([1]). Let $\mathrm{C}$ be a closed convex subset of a real Hilbert space $\mathrm{H}$ and sequence $\left\{\mathrm{x}_{\mathrm{n}}\right\}$ be bounded in $\mathrm{H}$. If

(i) $\omega_{w}\left(x_{n}\right) \subset C$;

(ii) $\lim _{\mathrm{n} \rightarrow \infty}\left\|x_{\mathrm{n}}-\mathrm{p}\right\|$ exists, $\forall \mathrm{p} \in \mathrm{C}$.

Then sequence $\left\{x_{n}\right\}$ converges weakly to a point in $\mathrm{C}$. 
Lemma 2.6 ([14]). Let $\mathrm{C}$ be a closed convex subset of a real Hilbert space $\mathrm{H}$ and sequence $\left\{\mathrm{x}_{\mathrm{n}}\right\}$ be bounded in $\mathrm{H}$. Let $\mathrm{q}=\mathrm{P}_{\mathrm{C}} \mathrm{x}, \mathrm{x} \in \mathrm{H}$. Assume

(i) $\omega_{w}\left(x_{n}\right) \subset C$;

(ii) $\left\|x_{\mathfrak{n}}-x\right\| \leqslant\|x-q\|, \forall n \in N$.

Then sequence $\left\{x_{n}\right\}$ converges strongly to $\mathrm{q}$.

Remark 2.7. We now give an example of a k-strictly asymptotically pseudocontractive mapping with nonempty set of fixed points.

Suppose that $H:=R$ and $C:=[-1,1] \subset R$. Let $T: C \rightarrow C$ be defined by

$$
\mathrm{T} x:=\left\{\begin{array}{l}
x, \quad x \in[-1,0) ; \\
\frac{1}{2} x, \quad x \in[0,1] .
\end{array}\right.
$$

Then we observe that $F(T)=[-1,0]$, and hence the set of the fixed points is nonempty.

Now, we show that $\mathrm{T}$ is a $\mathrm{k}$-strictly asymptotically pseudocontractive mapping. Suppose that $\mathrm{C}_{1}=$ $[-1,0)$ and $C_{2}=[0,1]$.

Case 1. If $x, y \in C_{1}$, then, $T^{n} x=x, T^{n} y=y$, we have that

$$
\left|T^{n} x-T^{n} y\right|=|x-y| \text {. }
$$

Case 2. If $x, y \in C_{2}$, then, $T^{n} x=\frac{1}{2^{n}} x, T^{n} y=\frac{1}{2^{n}} y$, we have that

$$
\left|T^{n} x-T^{n} y\right|=\frac{1}{2^{n}}|x-y| \text {. }
$$

Case 3. If $x \in C_{1}, y \in C_{2}$, then, $T^{n} x=x, T^{n} y=\frac{1}{2^{n}} y$, we have that

$$
\left|T^{n} x-T^{n} y\right|=\left|x-\frac{1}{2^{n}} y\right| \leqslant|x-y| .
$$

Case 4. If $x \in C_{2}, y \in C_{1}$, then, $T^{n} x=\frac{1}{2^{n}} x, T^{n} y=y$, we have that

$$
\left|T^{n} x-T^{n} y\right|=\left|\frac{1}{2^{n}} x-y\right| \leqslant|x-y| .
$$

Therefore, from (2.4), (2.5), (2.6), and (2.7), it is obvious that $\mathrm{T}$ is an asymptotically nonexpansive mapping with $k_{n} \equiv 1$, then $T$ is a $k$-strictly asymptotically pseudocontractive mapping for any $k \in[0,1)$.

\section{Main results}

In this section, we first prove two weak convergence theorems via two kinds of iteration schemes for finding a common element of the set of solutions of equilibrium problems (GMEP and GEP) and the set of common fixed points of a finite family of k-strictly asymptotically pseudo-contractions in Hilbert spaces. Two strong convergence theorems are also established based on the hybrid algorithm.

\subsection{Weak convergence theorems}

Theorem 3.1. Let $\mathrm{C}$ be a nonempty closed convex subset of an infinite dimensional real Hilbert space $\mathrm{H}$ and let $\left\{\mathrm{T}_{i}\right\}_{1 \leqslant i \leqslant N}$ be a finite family of $\left\{\mathrm{s}_{i}\right\}_{1 \leqslant i \leqslant N}$-strictly asymptotically pseudo-contractive mappings with sequence $\left\{s_{i}\right\} \subseteq[0,1)$ and $\left\{k_{n, i}\right\} \subseteq[1, \infty)$ such that $\lim _{n \rightarrow \infty} k_{n, i}=1$ and $\sum_{n=0}^{\infty}\left(k_{n, i}-1\right)<\infty$. Let $\varphi: C \rightarrow R$ be a proper lower semi-continuous and convex functional and let $\mathrm{B}: \mathrm{C} \rightarrow \mathrm{H}$ be a continuous and monotone mapping. Assume that $\Theta: C \times C \rightarrow R$ satisfies (A1)-(A4), $s=\max \left\{s_{i}: 1 \leqslant i \leqslant N\right\},\left\{k_{n}\right\}=\max \left\{k_{n, i}: 1 \leqslant i \leqslant N\right\}$ and $\Gamma=\bigcap_{i=1}^{\mathrm{N}} \mathrm{F}\left(\mathrm{T}_{i}\right) \cap \operatorname{GMEP}(\Theta, \mathrm{B}, \varphi) \neq \emptyset$. Let $\left\{x_{n}\right\}$ be a sequence generated by the following algorithm: 


$$
\left\{\begin{array}{l}
x_{0} \in C \text { chosen arbitrarily, } \\
y_{n-1}=\beta_{n-1} x_{n-1}+\left(1-\beta_{n-1}\right) T_{i(n)}^{h(n)} x_{n-1}, \\
z_{n-1}=\alpha_{n-1} x_{n-1}+\left(1-\alpha_{n-1}\right) T_{i(n)}^{h(n)} y_{n-1}, \\
x_{n} \in C \text { such that } \\
\quad \Theta\left(x_{n}, y\right)+\left\langle B x_{n}, y-x_{n}\right\rangle+\varphi(y)-\varphi\left(x_{n}\right)+\frac{1}{r_{n-1}}\left\langle y-x_{n}, x_{n}-z_{n-1}\right\rangle \geqslant 0, \forall y \in C, n \geqslant 1,
\end{array}\right.
$$

where $\left\{\alpha_{n}\right\},\left\{\beta_{n}\right\} \subset(0,1)$ and $\left\{r_{n}\right\}$ satisfying the following conditions:

(i) $\frac{\alpha_{n}}{\beta_{n}} \geqslant s, \forall n \geqslant 0$;

(ii) $\liminf _{n \rightarrow \infty} r_{n}>0, \forall n \geqslant 0$;

(iii) $\liminf _{\mathrm{n} \rightarrow \infty} \mathrm{g}_{\mathrm{n}-1}>0, \forall \mathrm{n} \geqslant 1$,

where $g_{n-1}=\left(1-\alpha_{n-1}\right)\left(1-\beta_{n-1}\right)\left[k_{h(n)}^{2}\left(\beta_{n-1}-s\right)-\left(1-\beta_{n-1}\right)^{2} L^{2} s+\beta_{n-1} s\right]$.

Then sequence $\left\{x_{n}\right\}$ converges weakly to a point in $\Gamma$.

Proof. Our proof is divided into the following three steps.

Step 1. We prove that $\lim _{n \rightarrow \infty}\left\|x_{n}-p\right\|$ exists, for all $p \in \Gamma$.

From (3.1) and (2.1), we have that

$$
\begin{aligned}
\left\|z_{n-1}-p\right\|^{2}= & \left\|\alpha_{n-1} x_{n-1}+\left(1-\alpha_{n-1}\right) T_{i(n)}^{h(n)} y_{n-1}-p\right\|^{2} \\
= & \alpha_{n-1}\left\|x_{n-1}-p\right\|^{2}+\left(1-\alpha_{n-1}\right)\left\|T_{i(n)}^{h(n)} y_{n-1}-p\right\|^{2} \\
& -\alpha_{n-1}\left(1-\alpha_{n-1}\right)\left\|x_{n-1}-T_{i(n)}^{h(n)} y_{n-1}\right\|^{2},
\end{aligned}
$$

and

$$
\begin{aligned}
\left\|y_{n-1}-p\right\|^{2}= & \left\|\beta_{n-1} x_{n-1}+\left(1-\beta_{n-1}\right) T_{i(n)}^{h(n)} x_{n-1}-p\right\|^{2} \\
= & \beta_{n-1}\left\|x_{n-1}-p\right\|^{2}+\left(1-\beta_{n-1}\right)\left\|T_{i(n)}^{h(n)} x_{n-1}-p\right\|^{2} \\
& -\beta_{n-1}\left(1-\beta_{n-1}\right)\left\|x_{n-1}-T_{i(n)}^{h(n)} x_{n-1}\right\|^{2} .
\end{aligned}
$$

Since that $T_{i}$ is a $s_{i}$-strictly asymptotically pseudo-contractive mapping, where $i \in\{1,2, \ldots, N\}$, one has

$$
\left\|T_{i(n)}^{h(n)} y_{n-1}-p\right\|^{2} \leqslant k_{h(n)}^{2}\left\|y_{n-1}-p\right\|^{2}+s\left\|y_{n-1}-T_{i(n)}^{h(n)} y_{n-1}\right\|^{2},
$$

and

$$
\left\|T_{i(n)}^{h(n)} x_{n-1}-p\right\|^{2} \leqslant k_{h(n)}^{2}\left\|x_{n-1}-p\right\|^{2}+s\left\|x_{n-1}-T_{i(n)}^{h(n)} x_{n-1}\right\|^{2} .
$$

Substituting (3.5) into (3.3), we obtain that

$$
\begin{aligned}
\left\|y_{n-1}-p\right\|^{2} \leqslant & \beta_{n-1}\left\|x_{n-1}-p\right\|^{2}+\left(1-\beta_{n-1}\right)\left(k_{h(n)}^{2}\left\|x_{n-1}-p\right\|^{2}\right. \\
& \left.+s\left\|x_{n-1}-T_{i(n)}^{h(n)} x_{n-1}\right\|^{2}\right)-\beta_{n-1}\left(1-\beta_{n-1}\right)\left\|x_{n-1}-T_{i(n)}^{h(n)} x_{n-1}\right\|^{2} \\
\leqslant & k_{h(n)}^{2}\left\|x_{n-1}-p\right\|^{2}-\left(\beta_{n-1}-s\right)\left(1-\beta_{n-1}\right)\left\|x_{n-1}-T_{i(n)}^{h(n)} x_{n-1}\right\|^{2} .
\end{aligned}
$$

Observe that

$$
\begin{aligned}
\left\|y_{n-1}-T_{i(n)}^{h(n)} y_{n-1}\right\|^{2}= & \left\|\beta_{n-1} x_{n-1}+\left(1-\beta_{n-1}\right) T_{i(n)}^{h(n)} x_{n-1}-T_{i(n)}^{h(n)} y_{n-1}\right\|^{2} \\
= & \beta_{n-1}\left\|x_{n-1}-T_{i(n)}^{h(n)} y_{n-1}\right\|^{2}+\left(1-\beta_{n-1}\right) \| T_{i(n)}^{h(n)} x_{n-1} \\
& -T_{i(n)}^{h(n)} y_{n-1}\left\|^{2}-\beta_{n-1}\left(1-\beta_{n-1}\right)\right\| x_{n-1}-T_{i(n)}^{h(n)} x_{n-1} \|^{2} \\
\leqslant & \beta_{n-1}\left\|x_{n-1}-T_{i(n)}^{h(n)} y_{n-1}\right\|^{2}+\left(1-\beta_{n}\right) L^{2}\left\|x_{n-1}-y_{n-1}\right\|^{2} \\
& -\beta_{n-1}\left(1-\beta_{n-1}\right) \mid x_{n-1}-T_{i(n)}^{h(n)} x_{n-1} \|^{2} \\
\leqslant & \beta_{n-1}\left\|x_{n-1}-T_{i(n)}^{h(n)} y_{n-1}\right\|^{2}+\left(1-\beta_{n}\right)^{3} L^{2} \| x_{n-1} \\
& -T_{i(n)}^{h(n)} x_{n-1}\left\|^{2}-\beta_{n-1}\left(1-\beta_{n-1}\right)\right\| x_{n-1}-T_{i(n)}^{h(n)} x_{n-1} \|^{2} .
\end{aligned}
$$


Substituting (3.6), (3.7) into (3.4), we get that

$$
\begin{aligned}
\left\|T_{i(n)}^{h(n)} y_{n-1}-p\right\|^{2} \leqslant & k_{h(n)}^{2}\left[k_{h(n)}^{2}\left\|x_{n-1}-p\right\|^{2}-\left(\beta_{n-1}-s\right)\left(1-\beta_{n-1}\right) \| x_{n-1}\right. \\
& \left.-T_{i(n)}^{h(n)} x_{n-1} \|^{2}\right]+s\left[\beta_{n-1}\left\|x_{n-1}-T_{i(n)}^{h(n)} y_{n-1}\right\|^{2}\right. \\
& +\left(1-\beta_{n}\right)^{3} L^{2}\left\|x_{n-1}-T_{i(n)}^{h(n)} x_{n-1}\right\|^{2} \\
& \left.-\beta_{n-1}\left(1-\beta_{n-1}\right)\left\|x_{n-1}-T_{i(n)}^{h(n)} x_{n-1}\right\|^{2}\right] \\
= & k_{h(n)}^{4}\left\|x_{n-1}-p\right\|^{2}+s \beta_{n-1}\left\|x_{n-1}-T_{i(n)}^{h(n)} y_{n-1}\right\|^{2} \\
& -\left(1-\beta_{n-1}\right)\left[k_{h(n)}^{2}\left(\beta_{n-1}-s\right)-\left(1-\beta_{n-1}\right)^{2} L^{2} s\right. \\
& \left.+s \beta_{n-1}\right]\left\|x_{n-1}-T_{i(n)}^{h(n)} x_{n-1}\right\|^{2} .
\end{aligned}
$$

Substituting (3.8) into (3.2), and combining with condition (i), we obtain that

$$
\begin{aligned}
\left\|z_{n-1}-p\right\|^{2}= & \alpha_{n-1}\left\|x_{n-1}-p\right\|^{2}+\left(1-\alpha_{n-1}\right)\left[k_{h(n)}^{4}\left\|x_{n-1}-p\right\|^{2}\right. \\
& +s \beta_{n-1}\left\|x_{n-1}-T_{i(n)}^{h(n)} y_{n-1}\right\|^{2}-\left(1-\beta_{n-1}\right)\left[k_{h(n)}^{2}\left(\beta_{n-1}-s\right)\right. \\
& \left.\left.-\left(1-\beta_{n-1}\right)^{2} L^{2} s+s \beta_{n-1}\right]\left\|x_{n-1}-T_{i(n)}^{h(n)} x_{n-1}\right\|^{2}\right] \\
& -\alpha_{n-1}\left(1-\alpha_{n-1}\right)\left\|x_{n-1}-T_{i(n)}^{h(n)} y_{n-1}\right\|^{2} \\
\leqslant & k_{h(n)}^{4}\left\|x_{n-1}-p\right\|^{2}-\left(1-\alpha_{n-1}\right)\left(\alpha_{n-1}-\beta_{n-1} s\right) \\
& \times\left\|x_{n-1}-T_{i(n)}^{h(n)} y_{n-1}\right\|^{2}-g_{n-1}\left\|x_{n-1}-T_{i(n)}^{h(n)} x_{n-1}\right\|^{2} \\
\leqslant & k_{h(n)}^{4}\left\|x_{n-1}-p\right\|^{2}-g_{n-1}\left\|x_{n-1}-T_{i(n)}^{h(n)} x_{n-1}\right\|^{2} .
\end{aligned}
$$

Since $x_{n}=K_{r_{n-1}} z_{n-1}$, it follows from Lemma 2.1 and condition (iii) that

$$
\begin{aligned}
\left\|x_{n}-p\right\|^{2} & =\left\|K_{r_{n-1}} z_{n-1}-p\right\|^{2} \\
& \leqslant\left\|z_{n-1}-p\right\|^{2} \\
& \leqslant k_{h(n)}^{4}\left\|x_{n-1}-p\right\|^{2} \\
& =\left(1+k_{h(n)}^{4}-1\right)\left\|x_{n-1}-p\right\|^{2} .
\end{aligned}
$$

From condition $\sum_{n=0}^{\infty}\left(k_{n, i}-1\right)<\infty$, we have $\sum_{n=0}^{\infty}\left(k_{n}-1\right)<\infty$. Hence $\sum_{n=0}^{\infty}\left(k_{h(n)}^{4}-1\right)<\infty$. Again by Lemma 2.2, we have that $\lim _{n \rightarrow \infty}\left\|x_{n}-p\right\|$ exists. So, $\left\{\left\|x_{n}-p\right\|\right\}$ is bounded, this implies that $\left\{x_{n}\right\}$ is bounded.

Step 2. We prove that $\omega_{w}\left(x_{n}\right) \subseteq \Gamma$.

First, we prove that $\omega_{w}\left(x_{n}\right) \subseteq \bigcap_{l=1}^{N} F\left(T_{l}\right)$. In fact, we only prove that

$$
\lim _{n \rightarrow \infty}\left\|x_{n}-T_{l} x_{n}\right\| \rightarrow 0, \quad \forall l \in\{1,2, \ldots, N\} .
$$

It follows from (3.9) that

$$
g_{n-1}\left\|x_{n-1}-T_{i(n)}^{h(n)} x_{n-1}\right\|^{2} \leqslant k_{h(n)}^{4}\left\|x_{n-1}-p\right\|^{2}-\left\|x_{n}-p\right\|^{2} .
$$

From the fact that $\liminf _{n \rightarrow \infty} g_{n-1}>0, k_{h(n)} \rightarrow 1$ and $\lim _{n \rightarrow \infty}\left\|x_{n}-p\right\|$ exists, we obtain that

$$
\left\|x_{n-1}-T_{i(n)}^{h(n)} x_{n-1}\right\| \rightarrow 0, \quad(n \rightarrow \infty) .
$$


From (3.1) and Lemma 2.3, we have that

$$
\begin{aligned}
\left\|z_{n-1}-x_{n-1}\right\| & =\left\|\alpha_{n-1} x_{n-1}+\left(1-\alpha_{n-1}\right) T_{i(n)}^{h(n)} y_{n-1}-x_{n-1}\right\| \\
& =\left(1-\alpha_{n-1}\right)\left\|T_{i(n)}^{h(n)} y_{n-1}-x_{n-1}\right\| \\
& \leqslant\left(1-\alpha_{n-1}\right)\left[\left\|T_{i(n)}^{h(n)} y_{n-1}-T_{i(n)}^{h(n)} x_{n-1}\right\|+\left\|T_{i(n)}^{h(n)} x_{n-1}-x_{n-1}\right\|\right] \\
& \leqslant\left(1-\alpha_{n-1}\right)\left[L\left\|y_{n-1}-x_{n-1}\right\|+\left\|T_{i(n)}^{h(n)} x_{n-1}-x_{n-1}\right\|\right] \\
& =\left(1-\alpha_{n-1}\right)\left[\left(1-\beta_{n-1}\right) L+1\right]\left\|T_{i(n)}^{h(n)} x_{n-1}-x_{n-1}\right\| \rightarrow 0 .
\end{aligned}
$$

From Lemma 2.1, we get that

$$
\begin{aligned}
\left\|x_{n}-p\right\|^{2} & =\left\|K_{r_{n-1}} z_{n-1}-p\right\|^{2} \\
& \leqslant\left\langle x_{n}-p, z_{n-1}-p\right\rangle \\
& =\frac{1}{2}\left(\left\|z_{n-1}-p\right\|^{2}+\left\|x_{n}-p\right\|^{2}-\left\|z_{n-1}-x_{n}\right\|^{2}\right) .
\end{aligned}
$$

It follows that

$$
\begin{aligned}
\left\|z_{n-1}-x_{n}\right\|^{2} & \leqslant\left\|z_{n-1}-p\right\|^{2}-\left\|x_{n}-p\right\|^{2} \\
& \leqslant k_{h(n)}^{4}\left\|x_{n-1}-p\right\|^{2}-\left\|x_{n}-p\right\|^{2} \rightarrow 0 .
\end{aligned}
$$

Hence, we have

$$
\left\|x_{n}-x_{n-1}\right\| \leqslant\left\|x_{n}-z_{n-1}\right\|+\left\|z_{n-1}-x_{n-1}\right\| \rightarrow 0, \quad(n \rightarrow \infty) .
$$

It is obvious that

$$
\lim _{n \rightarrow \infty}\left\|x_{n}-x_{n+j}\right\|=0, \quad \forall j \in\{1,2, \ldots, N\}
$$

Notice that

$$
\begin{aligned}
\left\|x_{n}-T_{n+j} x_{n}\right\| & \leqslant\left\|x_{n}-x_{n+j}\right\|+\left\|x_{n+j}-T_{n+j} x_{n+j}\right\|+\left\|T_{n+j} x_{n+j}-T_{n+j} x_{n}\right\| \\
& \leqslant(1+L)\left\|x_{n}-x_{n+j}\right\|+\left\|x_{n+j}-T_{n+j} x_{n+j}\right\|,
\end{aligned}
$$

and

$$
\begin{aligned}
\left\|x_{n}-T_{n} x_{n}\right\| & \leqslant\left\|x_{n}-x_{n-1}\right\|+\left\|x_{n-1}-T_{n} x_{n-1}\right\|+\left\|T_{n} x_{n-1}-T_{n} x_{n}\right\| \\
& \leqslant(1+L)\left\|x_{n}-x_{n-1}\right\|+\left\|x_{n-1}-T_{n} x_{n-1}\right\| .
\end{aligned}
$$

For all $n>N$, we can write $n=(h(n)-1) N+i(n)$, where $i(n) \in\{1,2, \ldots N\}$. Then,

$$
n-N=(h(n)-1-1) N+i(n)=(h(n-N)-1) N+i(n-N),
$$

that is, $h(n-N)=h(n)-1, i(n-N)=i(n)$. We can obtain that

$$
\begin{gathered}
\left\|x_{n-1}-T_{n} x_{n-1}\right\| \leqslant\left\|x_{n-1}-T_{i(n)}^{h(n)} x_{n-1}\right\|+\left\|T_{i(n)}^{h(n)} x_{n-1}-T_{n} x_{n-1}\right\| \\
\leqslant\left\|x_{n-1}-T_{i(n)}^{h(n)} x_{n-1}\right\|+\left\|T_{i(n)}^{h(n)} x_{n-1}-T_{i(n)} x_{n-1}\right\| \\
\leqslant\left\|x_{n-1}-T_{i(n)}^{h(n)} x_{n-1}\right\|+L\left\|T_{i(n)}^{h(n)-1} x_{n-1}-x_{n-1}\right\| \\
\leqslant\left\|x_{n-1}-T_{i(n)}^{h(n)} x_{n-1}\right\|+L\left(\left\|T_{i(n)}^{h(n)-1} x_{n-1}-T_{i(n-N)}^{h(n)-1} x_{n-N}\right\|\right. \\
\left.+\left\|T_{i(n-N)}^{h(n)-1} x_{n-N}-x_{(n-N)-1}\right\|+\left\|x_{(n-N)-1}-x_{n-1}\right\|\right), \\
\left\|T_{i(n)}^{h(n)-1} x_{n-1}-T_{i(n-N)}^{h(n)-1} x_{n-N}\right\|=\left\|T_{i(n)}^{h(n)-1} x_{n-1}-T_{i(n)}^{h(n)-1} x_{n-N}\right\| \\
\leqslant L\left\|x_{n-1}-x_{n-N}\right\|
\end{gathered}
$$


and

$$
\begin{aligned}
\left\|T_{i(n-N)}^{h(n)-1} x_{n-N}-x_{(n-N)-1}\right\| \leqslant & \left\|T_{i(n-N)}^{h(n)-1} x_{n-N}-T_{i(n-N)}^{h(n-N)} x_{(n-N)-1}\right\| \\
& +\left\|T_{i(n-N)}^{h(n-N)} x_{(n-N)-1}-x_{(n-N)-1}\right\| \\
\leqslant & \left\|T_{i(n-N)}^{h(n-N)} x_{(n-N)-1}-x_{(n-N)-1}\right\| \\
& +L\left\|x_{n-N}-x_{(n-N)-1}\right\| .
\end{aligned}
$$

Substituting (3.16) and (3.17) into (3.15), we have that

$$
\begin{aligned}
\left\|x_{n-1}-T_{n} x_{n-1}\right\| \leqslant & \left\|x_{n-1}-T_{i(n)}^{h(n)} x_{n-1}\right\|+L\left(L\left\|x_{n-1}-x_{n-N}\right\|\right. \\
& +L\left\|x_{n-N}-x_{(n-N)-1}\right\|+\| T_{i(n-N)}^{h(n)} x_{(n-N)-1} \\
& \left.-x_{(n-N)-1}\|+\| x_{(n-N)-1}-x_{n-1} \|\right) .
\end{aligned}
$$

From (3.11) and (3.12), we have that

$$
\lim _{n \rightarrow \infty}\left\|x_{n-1}-T_{n} x_{n-1}\right\|=0 .
$$

Substituting (3.18) into (3.14), we can obtain

$$
\lim _{n \rightarrow \infty}\left\|x_{n}-T_{n} x_{n}\right\|=0 .
$$

It follows from (3.12), (3.13) and (3.19) that

$$
\lim _{n \rightarrow \infty}\left\|x_{n}-T_{l} x_{n}\right\|=0, \quad \forall l \in\{1,2, \ldots, N\} .
$$

Since $\left\{x_{n}\right\}$ is bounded, there exists a subsequence $\left\{x_{n_{j}}\right\}$ of $\left\{x_{n}\right\}$ such that $\left\{x_{n_{j}}\right\} \rightarrow z$. Since $\left\|x_{n_{j}}-T_{l} x_{n_{j}}\right\| \rightarrow$ $0,(n \rightarrow \infty)$, from Lemma 2.3, we have $z \in F\left(T_{l}\right)$, for all $l \in\{1,2, \ldots, N\}$. That is, $\omega_{w}\left(x_{n}\right) \subseteq \bigcap_{l=1}^{N} F\left(T_{l}\right)$.

Next, we show $\omega_{w}\left(x_{n}\right) \subseteq \operatorname{GMEP}(\Theta, B, \varphi)$. From $\lim _{n \rightarrow \infty}\left\|z_{n-1}-x_{n}\right\|=0$ and condition (ii), we have that

$$
\lim _{n \rightarrow \infty} \frac{\left\|z_{n-1}-x_{n}\right\|}{r_{n-1}}=0
$$

Since $x_{n}=K_{r_{n-1}} z_{n-1}$, we also have

$$
\Omega\left(x_{n}, y\right)+\frac{1}{r_{n-1}}\left\langle y-x_{n}, x_{n}-z_{n-1}\right\rangle \geqslant 0, \quad \forall y \in C,
$$

where

$$
\Omega\left(x_{n}, y\right)=\Theta\left(x_{n}, y\right)+\left\langle B x_{n}, y-x_{n}\right\rangle+\varphi(y)-\varphi\left(x_{n}\right)
$$

It follows from (A2) that

$$
\frac{1}{r_{n-1}}\left\langle y-x_{n}, x_{n}-z_{n-1}\right\rangle \geqslant-\Omega\left(x_{n}, y\right) \geqslant \Omega\left(y, x_{n}\right), \quad \forall y \in C .
$$

Again from (A4) and $\lim _{n \rightarrow \infty} \frac{\left\|z_{n-1}-x_{n}\right\|}{r_{n-1}}=0$, we can obtain that

$$
\Omega(y, z) \leqslant 0, \quad \forall y \in C, \quad \forall z \in \omega_{w}\left(x_{n}\right) .
$$

Put $y_{t}=t y+(1-t) z$, for all $t \in(0,1), y \in C$ and $z \in \omega_{w}\left(x_{n}\right)$, then, $y_{t} \in C$. Therefore, $\Omega\left(y_{t}, z\right) \leqslant 0$. From (A1), we can obtain that $0=\Omega\left(y_{t}, y_{t}\right) \leqslant t \Omega\left(y_{t}, y\right)+(1-t) \Omega\left(y_{t}, z\right) \leqslant t \Omega\left(y_{t}, y\right)$. So, $\Omega\left(y_{t}, y\right) \geqslant 0$, for all $y \in C$. Taking $t \rightarrow 0$, we have $\Omega(z, y) \geqslant 0$, for all $y \in C$, then, $z \in \operatorname{GMEP}(\Theta, B, \varphi)$. That is, $\omega_{w}\left(x_{n}\right) \subseteq \operatorname{GMEP}(\Theta, \mathrm{B}, \varphi)$, and $\omega_{w}\left(x_{n}\right) \subseteq \Gamma$.

Step 3 . We prove that sequence $\left\{x_{n}\right\}$ converges weakly to a point in $\Gamma$.

Since $\omega_{w}\left(x_{n}\right) \subseteq \Gamma$ and $\lim _{n \rightarrow \infty}\left\|x_{n}-p\right\|$ exists, it follows from Lemma 2.5 that sequence $\left\{x_{n}\right\}$ converges weakly to a point in $\Gamma$. 
Theorem 3.2. Let $\mathrm{C}$ be a nonempty closed convex subset of an infinite dimensional real Hilbert space $\mathrm{H}$ and let $\left\{\mathrm{T}_{i}\right\}_{1 \leqslant i \leqslant N}$ be a finite family of $\left\{\mathrm{s}_{i}\right\}_{1 \leqslant i \leqslant N}$-strictly asymptotically pseudo-contractive mappings with sequence $\left\{s_{i}\right\} \subseteq[0,1)$ and $\left\{k_{n, i}\right\} \subseteq[1, \infty)$ such that $\lim _{n \rightarrow \infty} k_{n, i}=1$ and $\sum_{n=0}^{\infty}\left(k_{n, i}-1\right)<\infty$. Let $\mathrm{B}: \mathrm{C} \rightarrow \mathrm{H}$ be an $\alpha-$ inverse-strongly monotone mapping. Assume that $\Theta: \mathrm{C} \times \mathrm{C} \rightarrow \mathrm{R}$ satisfies (A1)-(A4), $\mathrm{s}=\max \left\{\mathrm{s}_{\mathrm{i}}: 1 \leqslant i \leqslant \mathrm{~N}\right\}$, $\left\{k_{n}\right\}=\max \left\{k_{n, i}: 1 \leqslant i \leqslant N\right\}$ and $\Gamma=\bigcap_{i=1}^{N} F\left(T_{i}\right) \bigcap \operatorname{GEP}(\Theta, B) \neq \emptyset$. Let $\left\{x_{n}\right\}$ be a sequence generated by the following algorithm:

$$
\left\{\begin{array}{l}
x_{0} \in C \text { chosen arbitrarily, } \\
y_{n-1}=\beta_{n-1} x_{n-1}+\left(1-\beta_{n-1}\right) T_{i(n)}^{h(n)} x_{n-1}, \\
z_{n-1}=\alpha_{n-1} x_{n-1}+\left(1-\alpha_{n-1}\right) T_{i(n)}^{h(n)} y_{n-1}, \\
x_{n} \in C \text { such that } \\
\quad \Theta\left(x_{n}, y\right)+\left\langle B z_{n-1}, y-x_{n}\right\rangle+\frac{1}{r_{n-1}}\left\langle y-x_{n}, x_{n}-z_{n-1}\right\rangle \geqslant 0, \quad \forall y \in C, n \geqslant 1,
\end{array}\right.
$$

where $\left\{\alpha_{n}\right\},\left\{\beta_{n}\right\} \subset(0,1)$ and $\left\{r_{n}\right\}$ satisfying the following conditions:

(i) $\frac{\alpha_{n}}{\beta_{n}} \geqslant s, \forall n \geqslant 0$;

(ii) $\mathrm{r}_{\mathrm{n}} \in[\mathrm{a}, \mathrm{b}]$ for some $0<\mathrm{a}<\mathrm{b}<2 \alpha, \forall \mathrm{n} \geqslant 0$;

(iii) $\liminf _{n \rightarrow \infty} g_{n-1}>0$,

where $g_{n-1}=\left(1-\alpha_{n-1}\right)\left(1-\beta_{n-1}\right)\left[k_{h(n)}^{2}\left(\beta_{n-1}-s\right)-\left(1-\beta_{n-1}\right)^{2} L^{2} s+\beta_{n-1} s\right]$, for all $n \geqslant 1$.

Then sequence $\left\{x_{n}\right\}$ converges weakly to a point in $\Gamma$.

Proof. Our proof is divided into the following steps.

Step 1. We prove that $\lim _{n \rightarrow \infty}\left\|x_{n}-p\right\|$ exists, for all $p \in \Gamma$.

From algorithm (3.20), we can obtain that $x_{n}=T_{r_{n-1}}\left(z_{n-1}-r_{n-1} B z_{n-1}\right)$. Again from Lemma 2.1 and (2.3), we have that

$$
\begin{aligned}
\left\|x_{n}-p\right\|^{2} & =\left\|T_{r_{n-1}}\left(z_{n-1}-r_{n-1} B z_{n-1}\right)-T_{r_{n-1}}\left(p-r_{n-1} B p\right)\right\|^{2} \\
& \leqslant\left\|z_{n-1}-r_{n-1} B z_{n-1}-\left(p-r_{n-1} B p\right)\right\|^{2} \\
& \leqslant\left\|z_{n-1}-p\right\|^{2}+r_{n-1}\left(r_{n-1}-2 \alpha\right)\left\|B z_{n-1}-B p\right\|^{2} .
\end{aligned}
$$

Similar to the first step of Theorem 3.1, we find from conditions (ii) and (iii) that

$$
\begin{aligned}
\left\|x_{n}-p\right\|^{2} \leqslant & \left\|z_{n-1}-p\right\|^{2}+r_{n-1}\left(r_{n-1}-2 \alpha\right)\left\|B z_{n-1}-B p\right\|^{2} \\
\leqslant & k_{h(n)}^{4}\left\|x_{n-1}-p\right\|^{2}-g_{n-1}\left\|x_{n-1}-T_{i(n)}^{h(n)} x_{n-1}\right\|^{2} \\
& +r_{n-1}\left(r_{n-1}-2 \alpha\right)\left\|B z_{n-1}-B p\right\|^{2} \\
\leqslant & \left(1+k_{h(n)}^{4}-1\right)\left\|x_{n-1}-p\right\|^{2} .
\end{aligned}
$$

Since $\sum_{n=0}^{\infty}\left(k_{n, i}-1\right)<\infty$, we have $\sum_{n=0}^{\infty}\left(k_{n}-1\right)<\infty$. Hence $\sum_{n=0}^{\infty}\left(k_{h_{n}}^{4}-1\right)<\infty$. Again by Lemma 2.2, we have that $\lim _{n \rightarrow \infty}\left\|x_{n}-p\right\|$ exists. So, $\left\{\left\|x_{n}-p\right\|\right\}$ is bounded, this implies that $\left\{x_{n}\right\}$ is bounded.

Step 2. We prove that $\omega_{w}\left(x_{n}\right) \subseteq \Gamma$. The difference between Theorem 3.1 and Theorem 3.2 is the proof of $\lim _{n \rightarrow \infty}\left\|x_{n}-T_{l} x_{n}\right\| \rightarrow 0$, for all $l \in\{1,2, \ldots, N\}$ and $\omega_{w}\left(x_{n}\right) \subseteq \operatorname{GEP}(\Theta, B)$. From (3.21), we have that

$$
g_{n-1}\left\|x_{n-1}-T_{i(n)}^{h(n)} x_{n-1}\right\|^{2} \leqslant k_{h(n)}^{4}\left\|x_{n-1}-p\right\|^{2}-\left\|x_{n}-p\right\|^{2},
$$

and

$$
r_{n-1}\left(2 \alpha-r_{n-1}\right)\left\|B z_{n-1}-B p\right\|^{2} \leqslant k_{h(n)}^{4}\left\|x_{n-1}-p\right\|^{2}-\left\|x_{n}-p\right\|^{2} .
$$

Again from conditions (ii) and (iii), we have that

$$
\lim _{n \rightarrow \infty}\left\|x_{n-1}-T_{i(n)}^{h(n)} x_{n-1}\right\|=0,
$$


and

$$
\lim _{n \rightarrow \infty}\left\|\mathrm{B} z_{n-1}-\mathrm{Bp}\right\|=0
$$

Observe that

$$
\begin{aligned}
\left\|z_{n-1}-x_{n-1}\right\|= & \left\|\alpha_{n-1} x_{n-1}+\left(1-\alpha_{n-1}\right) T_{i(n)}^{h(n)} y_{n-1}-x_{n-1}\right\| \\
= & \left(1-\alpha_{n-1}\right)\left\|T_{i(n)}^{h(n)} y_{n-1}-x_{n-1}\right\| \\
\leqslant & \left(1-\alpha_{n-1}\right)\left\|T_{i(n)}^{h(n)} y_{n-1}-T_{i(n)}^{h(n)} x_{n-1}\right\| \\
& +\left\|T_{i(n)}^{h(n)} x_{n-1}-x_{n-1}\right\| \\
\leqslant & \left(1-\alpha_{n-1}\right)\left[L\left\|y_{n-1}-x_{n-1}\right\|\right. \\
& \left.+\left\|T_{i(n)}^{h(n)} x_{n-1}-x_{n-1}\right\|\right] \\
= & \left(1-\alpha_{n-1}\right)\left[\left(1-\beta_{n-1}\right) L+1\right]\left\|T_{i(n)}^{h(n)} x_{n-1}-x_{n-1}\right\| \\
\rightarrow & 0 .
\end{aligned}
$$

From (3.20) and Lemma 2.1, we have that

$$
\begin{aligned}
\left\|x_{n}-p\right\|^{2}= & \left\|T_{r_{n-1}}\left(z_{n-1}-r_{n-1} B z_{n-1}\right)-T_{r_{n-1}}\left(p-r_{n-1} B p\right)\right\|^{2} \\
\leqslant & \left\langle z_{n-1}-r_{n-1} B z_{n-1}-\left(p-r_{n-1} B p\right), x_{n}-p\right\rangle \\
= & \frac{1}{2}\left(\left\|\left(z_{n-1}-r_{n-1} B z_{n-1}\right)-\left(p-r_{n-1} B p\right)\right\|^{2}\right)+\left\|x_{n}-p\right\|^{2} \\
& -\left\|\left(z_{n-1}-r_{n-1} B z_{n-1}\right)-\left(p-r_{n-1} B p\right)-\left(x_{n}-p\right)\right\|^{2} \\
\leqslant & \frac{1}{2}\left(\left\|z_{n-1}-p\right\|^{2}+\left\|x_{n}-p\right\|^{2}-\left\|\left(z_{n-1}-x_{n}\right)-r_{n-1}\left(B z_{n-1}-B p\right)\right\|^{2}\right) \\
= & \frac{1}{2}\left(\left\|z_{n-1}-p\right\|^{2}+\left\|x_{n}-p\right\|^{2}-\left\|z_{n-1}-x_{n}\right\|^{2}-r_{n-1}^{2}\left\|B z_{n-1}-B p\right\|^{2}\right. \\
& \left.+2 r_{n-1}\left\langle z_{n-1}-x_{n}, B z_{n-1}-B p\right\rangle\right) .
\end{aligned}
$$

It follows that

$$
\begin{aligned}
\left\|x_{n}-p\right\|^{2} \leqslant & \left\|z_{n-1}-p\right\|^{2}-\left\|z_{n-1}-x_{n}\right\|^{2}-r_{n-1}^{2}\left\|B z_{n-1}-B p\right\|^{2} \\
& +2 r_{n-1}\left\langle z_{n-1}-x_{n}, B z_{n-1}-B p\right\rangle \\
\leqslant & k_{h(n)}^{4}\left\|x_{n-1}-p\right\|^{2}-\left\|z_{n-1}-x_{n}\right\|^{2}-r_{n-1}^{2}\left\|B z_{n-1}-B p\right\|^{2} \\
& +2 r_{n-1}\left\langle z_{n-1}-x_{n}, B z_{n-1}-B p\right\rangle .
\end{aligned}
$$

So, we have

$$
\begin{aligned}
\left\|z_{n-1}-x_{n}\right\|^{2} \leqslant & \left\|x_{n-1}-p\right\|^{2}-\left\|x_{n}-p\right\|^{2}+\left(k_{h(n)}^{4}-1\right)\left\|x_{n-1}-p\right\|^{2} \\
& -r_{n-1}^{2}\left\|B z_{n-1}-B p\right\|^{2}+2 r_{n-1}\left\langle z_{n-1}-x_{n}, B z_{n-1}-B p\right\rangle .
\end{aligned}
$$

Since $\lim _{n \rightarrow \infty} k_{n}=1, \lim _{n \rightarrow \infty}\left\|B z_{n-1}-B p\right\|=0$ and $\lim _{n \rightarrow \infty}\left\|x_{n}-p\right\|$ exists, we obtain that

$$
\lim _{n \rightarrow \infty}\left\|z_{n-1}-x_{n}\right\|=0 \text {. }
$$

Therefore,

$$
\left\|x_{n}-x_{n-1}\right\| \leqslant\left\|x_{n}-z_{n-1}\right\|+\left\|z_{n-1}-x_{n-1}\right\| \rightarrow 0 .
$$

The remaining is the same to the proof of $\lim _{n \rightarrow \infty}\left\|x_{n}-T_{l} x_{n}\right\| \rightarrow 0$, for all $l \in\{1,2, \ldots, N\}$.

Next, we prove that $\omega_{w}\left(x_{n}\right) \subseteq \operatorname{GEP}(\Theta, B)$. Since $x_{n}=T_{r_{n-1}}\left(z_{n-1}-r_{n-1} A z_{n-1}\right)$, we have

$$
\Theta\left(x_{n}, y\right)+\left\langle B z_{n-1}, y-x_{n}\right\rangle+\frac{1}{r_{n-1}}\left\langle y-x_{n}, x_{n}-z_{n-1}\right\rangle \geqslant 0, \quad \forall y \in C .
$$


It follows from (A2) that

$$
\left\langle\mathrm{B} z_{n-1}, y-x_{n}\right\rangle+\frac{1}{r_{n-1}}\left\langle y-x_{n}, x_{n}-z_{n-1}\right\rangle \geqslant \Theta\left(y, x_{n}\right) .
$$

Let $y_{t}=t y+(1-t) z$, where $y \in C, z \in \omega_{w}\left(x_{n}\right)$ and $t \in(0,1)$, it is easy to see that $y_{t} \in C$. Combining with (3.23), we can obtain that

$$
\begin{aligned}
\left\langle B y_{t}, y_{t}-x_{n}\right\rangle \geqslant & \left\langle B y_{t}, y_{t}-x_{n}\right\rangle-\left\langle B z_{n-1}, y_{t}-x_{n}\right\rangle \\
& -\frac{1}{r_{n-1}}\left\langle y_{t}-x_{n}, x_{n}-z_{n-1}\right\rangle+\Theta\left(y_{t}, x_{n}\right) \\
\geqslant & \left\langle B y_{t}-B x_{n}, y_{t}-x_{n}\right\rangle+\left\langle B x_{n}-B z_{n-1}, y_{t}-x_{n}\right\rangle \\
& -\left\langle y_{t}-x_{n}, \frac{x_{n}-z_{n-1}}{r_{n-1}}\right\rangle+\Theta\left(y_{t}, x_{n}\right) .
\end{aligned}
$$

It follows from $\lim _{n \rightarrow \infty}\left\|z_{n-1}-x_{n}\right\|=0$ that $\lim _{n \rightarrow \infty} \frac{\left\|z_{n-1}-x_{n}\right\|}{r_{n-1}}=0$. On the other hand, we have $\left\|B x_{n}-B z_{n-1}\right\| \rightarrow 0$. Again by monotonicity of $B$, we obtain that $\left\langle B y_{t}-B x_{n}, y_{t}-x_{n}\right\rangle \geqslant 0$. Then, replacing $n$ by $n_{k}$ and letting $k \rightarrow \infty$, from (A4), we can easily observe that

$$
\left\langle B y_{t}, y_{t}-z\right\rangle \geqslant \Theta\left(y_{t}, z\right) .
$$

Again combining with (A1) and (A4), we have

$$
\begin{aligned}
0 & =\Theta\left(y_{t}, y_{t}\right) \\
& \leqslant t \Theta\left(y_{t}, y\right)+(1-t) \Theta\left(y_{t}, z\right) \\
& \leqslant t \Theta\left(y_{t}, y\right)+(1-t) t\left\langle B y_{t}, y-z\right\rangle
\end{aligned}
$$

It follows that

$$
\Theta\left(y_{t}, y\right)+(1-t)\left\langle B y_{t}, y-z\right\rangle \geqslant 0
$$

Taking $t \rightarrow 0$, we obtain that

$$
\Theta(z, y)+\langle B z, y-z\rangle \geqslant 0, \quad \forall y \in C .
$$

Then $z \in \operatorname{GEP}(\Theta, \mathrm{B})$, that is, $\omega_{w}\left(x_{n}\right) \subseteq \operatorname{GEP}(\Theta, B)$. The remaining is the same to the proof of Theorem 3.1.

Remark 3.3. Since asymptotically nonexpansive mappings are 0 -strict asymptotically pseudo-contractions, therefore, we can obtain two kinds of weak convergence theorems for a finite family of asymptotically nonexpansive mappings by putting $s_{i}=0$, for all $i \in\{1,2, \ldots, N\}$ in Theorem 3.1 with the condition replaced of the following:

(i) $\liminf _{n \rightarrow \infty} r_{n}>0, \forall n \geqslant 0$;

(ii) $\left\{\alpha_{n}\right\} \subset[0,1),\left\{\beta_{n}\right\} \subset(0,1)$;

(iii) $\limsup _{n \rightarrow \infty} \alpha_{n}<1,0<\liminf _{n \rightarrow \infty} \beta_{n} \leqslant \limsup _{n \rightarrow \infty} \beta_{n}<1$,

and in Theorem 3.2 with the condition replaced of the following:

(i) $r_{n} \in[a, b]$ for some $0<a<b<2 \alpha, \forall n \geqslant 0$;

(ii) $\left\{\alpha_{n}\right\} \subset[0,1),\left\{\beta_{n}\right\} \subset(0,1)$;

(iii) $\limsup _{n \rightarrow \infty} \alpha_{n}<1,0<\liminf _{n \rightarrow \infty} \beta_{n} \leqslant \limsup _{n \rightarrow \infty} \beta_{n}<1$.

\subsection{Strong convergence theorems}

Theorem 3.4. Let $\mathrm{C}$ be a nonempty closed convex subset of an infinite dimensional real Hilbert space $\mathrm{H}$ and let $\left\{\mathrm{T}_{i}\right\}_{1 \leqslant i \leqslant N}$ be a finite family of $\left\{\mathrm{s}_{i}\right\}_{1 \leqslant i \leqslant N}$-strictly asymptotically pseudo-contractive mappings with sequence 
$\left\{s_{i}\right\} \subseteq[0,1)$ and $\left\{k_{n, i}\right\} \subseteq[1, \infty)$ such that $\lim _{n \rightarrow \infty} k_{n, i}=1$ and $\sum_{n=0}^{\infty}\left(k_{n, i}-1\right)<\infty$. Let $\varphi: C \rightarrow R$ be a proper lower semi-continuous and convex functional, and let $\mathrm{B}: \mathrm{C} \rightarrow \mathrm{H}$ be a continuous and monotone mapping. Assume that $\Theta: C \times C \rightarrow R$ satisfies (A1)-(A4), $s=\max \left\{s_{i}: 1 \leqslant i \leqslant N\right\},\left\{k_{n}\right\}=\max \left\{k_{n, i}: 1 \leqslant i \leqslant N\right\}$ and $\Gamma=\bigcap_{i=1}^{N} F\left(T_{i}\right) \cap \operatorname{GMEP}(\Theta, B, \varphi) \neq \emptyset$. Let $\left\{x_{n}\right\}$ be a sequence generated by the following algorithm:

$$
\left\{\begin{array}{l}
x_{0} \in C \text { chosen arbitrarily, } \\
y_{n-1}=\beta_{n-1} x_{n-1}+\left(1-\beta_{n-1}\right) T_{i(n)}^{h(n)} x_{n-1}, \\
z_{n-1}=\alpha_{n-1} x_{n-1}+\left(1-\alpha_{n-1}\right) T_{i(n)}^{h(n)} y_{n-1}, \\
u_{n-1} \in C, \text { such that for all } y \in C, \\
\quad \Theta\left(u_{n-1}, y\right)+\left\langle B u_{n-1}, y-u_{n-1}\right\rangle+\varphi(y)-\varphi\left(u_{n-1}\right)+\frac{1}{r_{n-1}}\left\langle y-u_{n-1}, u_{n-1}-z_{n-1}\right\rangle \geqslant 0, \\
C_{n-1}=\left\{v \in C:\left\|u_{n-1}-v\right\|^{2} \leqslant\left\|x_{n-1}-v\right\|^{2}+\theta_{n-1}-h_{n-1}\left\|x_{n-1}-T_{i(n)}^{h(n)} x_{n-1}\right\|^{2}\right\}, \\
Q_{n-1}=\left\{v \in C:\left\langle x_{0}-x_{n-1}, x_{n-1}-v\right\rangle \geqslant 0\right\} \\
x_{n}=P_{C_{n-1} \cap Q_{n-1}} x_{0}, \forall n \geqslant 1,
\end{array}\right.
$$

where $\theta_{n}=\left(k_{h(n)}^{4}-1\right) \rho_{n}, \rho_{n}=\sup \left\{\left\|x_{n}-p\right\|: p \in \Gamma\right\}<\infty$. When $\left\{\alpha_{n}\right\},\left\{\beta_{n}\right\} \subset(0,1)$ and $\left\{r_{n}\right\}$ satisfying the following conditions:

(i) $\frac{\alpha_{n}}{\beta_{n}} \geqslant s, \forall n \geqslant 0$;

(ii) $\liminf _{n \rightarrow \infty} r_{n}>0, \forall n \geqslant 0$;

(iii) $\liminf _{\mathrm{n} \rightarrow \infty} \mathrm{g}_{\mathrm{n}-1}>0, \forall \mathrm{n} \geqslant 1$,

where $g_{n-1}=\left(1-\alpha_{n-1}\right)\left(1-\beta_{n-1}\right)\left[k_{h(n)}^{2}\left(\beta_{n-1}-s\right)-\left(1-\beta_{n-1}\right)^{2} L^{2} s+\beta_{n-1} s\right]$.

Then sequence $\left\{x_{n}\right\}$ converges strongly to $\mathrm{P}_{\Gamma} \mathrm{x}_{0}$.

Proof. Our proof is divided into the following five steps.

Step 1. We show that $C_{n-1}$ and $Q_{n-1}$ are closed and convex for all $n \geqslant 1$.

From the definition of $C_{n-1}$ and $Q_{n-1}$, it is obvious that $C_{n-1}$ is closed and $Q_{n-1}$ is closed and convex. From Lemma 2.4, we know that $\mathrm{C}_{n-1}$ is also convex.

Step 2. We show that $\Gamma \subseteq \mathrm{C}_{n-1} \cap \mathrm{Q}_{n-1}$, for all $n \geqslant 1$.

The proof of $\Gamma \subseteq C_{n-1}$ is similar to the first step of Theorem 3.1, we only replace $x_{n}$ with $u_{n-1}$ in (3.9) and (3.10). Then, we can obtain for all $p \in \Gamma$,

$$
\begin{aligned}
\left\|u_{n-1}-p\right\|^{2} & \leqslant\left\|z_{n-1}-p\right\|^{2} \\
& \leqslant k_{h(n)}^{4}\left\|x_{n-1}-p\right\|^{2}-g_{n-1}\left\|x_{n-1}-T_{i(n)}^{h(n)} x_{n-1}\right\|^{2} \\
& \leqslant\left\|x_{n-1}-p\right\|^{2}+\theta_{n-1}-g_{n-1}\left\|x_{n-1}-T_{i(n)}^{h(n)} x_{n-1}\right\|^{2},
\end{aligned}
$$

that is, $\Gamma \subseteq \mathrm{C}_{n-1}$, for all $\mathrm{n} \geqslant 1$.

We prove that $\Gamma \subseteq \mathrm{Q}_{n-1}$, for all $n \geqslant 1$ by induction. We have $\Gamma \subseteq \mathrm{C}=\mathrm{Q}_{0}$. Suppose $\Gamma \subseteq \mathrm{Q}_{\mathrm{n}-1}$. Since $x_{n}=P_{C_{n-1} \cap Q_{n-1}} x_{0}$, we find from (2.2) that

$$
\left\langle x_{0}-x_{n}, x_{n}-u\right\rangle \geqslant 0, \quad \forall u \in C_{n-1} \bigcap Q_{n-1} .
$$

Since $\Gamma \subseteq \mathrm{C}_{\mathrm{n}-1} \cap \mathrm{Q}_{\mathrm{n}-1}$, we have $\Gamma \subseteq \mathrm{Q}_{\mathrm{n}}$.

Step 3. We prove that $\left\{x_{n}\right\}$ is bounded.

Since the definition of $\mathrm{Q}_{n-1}$, we know that $x_{n-1}=\mathrm{P}_{\mathrm{Q}_{n-1}} x_{0}$. Also, $x_{n} \in \mathrm{C}_{n-1} \cap \mathrm{Q}_{n-1} \subseteq \mathrm{Q}_{n-1}$. Then

$$
\left\|x_{n-1}-x_{0}\right\| \leqslant\left\|x_{n}-x_{0}\right\| \text {. }
$$

Therefore, the sequence $\left\{\left\|x_{n}-x_{0}\right\|\right\}$ is nondecreasing. Again since $\Gamma \subseteq Q_{n-1}$, for all $n \geqslant 1$. It follows that

$$
\left\|x_{n-1}-x_{0}\right\| \leqslant\left\|p-x_{0}\right\|, \quad \forall p \in \Gamma .
$$


So, we have $\lim _{n \rightarrow \infty}\left\|x_{n}-x_{0}\right\|$ exists, that is, $\left\{x_{n}\right\}$ is bounded.

Step 4. We prove that $\omega_{w}\left(x_{n}\right) \subseteq \Gamma$.

The difference between Theorem 3.4 and Theorem 3.1 is the proof of

$$
\lim _{n \rightarrow \infty}\left\|x_{n}-T_{l} x_{n}\right\| \rightarrow 0, \quad \forall l \in\{1,2, \ldots, N\},
$$

and $\omega_{w}\left(x_{n}\right) \subseteq \operatorname{GMEP}(\Theta, B, \varphi)$.

First, we show that $\lim _{n \rightarrow \infty}\left\|x_{n}-T_{l} x_{n}\right\| \rightarrow 0$, for all $l \in\{1,2, \ldots, N\}$. Obviously, we only need to prove that $\lim _{n \rightarrow \infty}\left\|x_{n}-x_{n-1}\right\|=0$ and $\lim _{n \rightarrow \infty}\left\|x_{n-1}-T_{i(n)}^{h(n)} x_{n-1}\right\|=0$. Since the definition of $Q_{n-1}$, we know that $x_{n-1}=P_{Q_{n-1}} x_{0}$. Considering $x_{n} \in C_{n-1} \cap Q_{n-1} \subseteq Q_{n-1}$, we can obtain that

$$
\left\langle x_{n}-x_{n-1}, x_{n-1}-x_{0}\right\rangle \geqslant 0 \text {. }
$$

From (2.2), we know that

$$
\begin{aligned}
\left\|x_{n}-x_{n-1}\right\|^{2}= & \left\|\left(x_{n}-x_{0}\right)-\left(x_{n-1}-x_{0}\right)\right\|^{2} \\
= & \left\|x_{n}-x_{0}\right\|^{2}-\left\|x_{n-1}-x_{0}\right\|^{2} \\
& -2\left\langle x_{n}-x_{n-1}, x_{n-1}-x_{0}\right\rangle \\
\leqslant & \left\|x_{n}-x_{0}\right\|^{2}-\left\|x_{n-1}-x_{0}\right\|^{2},
\end{aligned}
$$

which together with the existence of $\lim _{n \rightarrow \infty}\left\|x_{n}-x_{0}\right\|$, we get that

$$
\left\|x_{n}-x_{n-1}\right\| \rightarrow 0, \quad(n \rightarrow \infty) .
$$

By the definition of $C_{n-1}$, we have

$$
\left\|u_{n-1}-v\right\|^{2} \leqslant\left\|x_{n-1}-v\right\|^{2}+\theta_{n-1}, \quad \forall v \in C_{n-1} .
$$

Again since $x_{n}=P_{C_{n-1} \cap Q_{n-1}} x_{0} \in C_{n-1}$, we have

$$
\left\|u_{n-1}-x_{n}\right\|^{2} \leqslant\left\|x_{n-1}-x_{n}\right\|^{2}+\theta_{n} .
$$

Combining (3.25) and $\theta_{n} \rightarrow 0(n \rightarrow \infty)$, it is obvious that $\lim _{n \rightarrow \infty}\left\|u_{n-1}-x_{n}\right\|=0$.

$$
\left\|u_{n-1}-x_{n-1}\right\| \leqslant\left\|u_{n-1}-x_{n}\right\|+\left\|x_{n}-x_{n-1}\right\| \rightarrow 0, \quad(n \rightarrow \infty) .
$$

From the definition of $C_{n-1}$, we have

$$
g_{n-1}\left\|x_{n-1}-T_{i(n)}^{h(n)} x_{n-1}\right\|^{2} \leqslant\left\|x_{n-1}-p\right\|^{2}+\theta_{n}-\left\|u_{n-1}-p\right\|^{2} .
$$

From (3.26), $\theta_{n} \rightarrow 0(n \rightarrow \infty)$ and condition (iii), we can obtain

$$
\left\|x_{n-1}-T_{i(n)}^{h(n)} x_{n-1}\right\| \rightarrow 0, \quad(n \rightarrow \infty) .
$$

The remaining is the same to Theorem 3.1.

Next, we show that $\omega_{w}\left(x_{n}\right) \subseteq \operatorname{GMEP}(\Theta, B, \varphi)$.

We only need to prove that $\lim _{n \rightarrow \infty}\left\|u_{n-1}-z_{n-1}\right\|=0$, for all $n \geqslant 1$. From (3.22) and (3.26), we have

$$
\left\|u_{n-1}-z_{n-1}\right\| \leqslant\left\|u_{n-1}-x_{n-1}\right\|+\left\|x_{n-1}-z_{n-1}\right\| \rightarrow 0 .
$$

The remaining is the same to Theorem 3.1. Therefore, $\omega_{w}\left(x_{n}\right) \subseteq \Gamma$.

Step 5. We prove that $x_{n} \rightarrow x^{*}=P_{\Gamma} x_{0}$. $\mathrm{P}_{\Gamma} \mathrm{x}_{0}$.

Combining with (3.24) and lemma 2.6, we can obtain that sequence $\left\{x_{n}\right\}$ converges strongly to $x^{*}=$ 
Theorem 3.5. Let $\mathrm{C}$ be a nonempty closed convex subset of an infinite dimensional real Hilbert space $\mathrm{H}$ and let $\left\{\mathrm{T}_{i}\right\}_{1 \leqslant i \leqslant N}$ be a finite family of $\left\{\mathrm{s}_{i}\right\}_{1 \leqslant i \leqslant N}$-strictly asymptotically pseudo-contractive mappings with sequence $\left\{s_{i}\right\} \subseteq[0,1)$ and $\left\{k_{n, i}\right\} \subseteq[1, \infty)$ such that $\lim _{n \rightarrow \infty} k_{n, i}=1$ and $\sum_{n=0}^{\infty}\left(k_{n, i}-1\right)<\infty$. Let $B: C \rightarrow H$ be an $\alpha$-inverse-strongly monotone mapping. Assume that $\Theta: \mathrm{C} \times \mathrm{C} \rightarrow \mathrm{R}$ satisfies (A1)-(A4), $s=\max \left\{s_{i}: 1 \leqslant i \leqslant N\right\}$, $\left\{k_{n}\right\}=\max \left\{k_{n, i}: 1 \leqslant i \leqslant N\right\}$ and $\Gamma=\bigcap_{i=1}^{N} F\left(T_{i}\right) \bigcap \operatorname{GEP}(\Theta, B) \neq \emptyset$. Let $\left\{x_{n}\right\}$ be a sequence generated by the following algorithm:

$$
\left\{\begin{array}{l}
x_{0} \in C \text { chosen arbitrarily, } \\
y_{n-1}=\beta_{n-1} x_{n-1}+\left(1-\beta_{n-1}\right) T_{i(n)}^{h(n)} x_{n-1}, \\
z_{n-1}=\alpha_{n-1} x_{n-1}+\left(1-\alpha_{n-1}\right) T_{i(n)}^{h(n)} y_{n-1}, \\
u_{n-1} \in C, \text { such that for all } y \in C, \\
\quad \Theta\left(u_{n-1}, y\right)+\left\langle B z_{n-1}, y-u_{n-1}\right\rangle+\frac{1}{r_{n-1}}\left\langle y-u_{n-1}, u_{n-1}-z_{n-1}\right\rangle \geqslant 0, \quad \forall y \in C, \\
C_{n}=\left\{v \in C:\left\|u_{n-1}-v\right\|^{2} \leqslant\left\|x_{n-1}-v\right\|^{2}+\theta_{n-1}-h_{n-1}\left\|x_{n-1}-T_{i(n)}^{h(n)} x_{n-1}\right\|^{2}\right\}, \\
Q_{n}=\left\{v \in C:\left\langle x_{0}-x_{n-1}, x_{n-1}-v\right\rangle \geqslant 0\right\}, \\
x_{n}=P_{C_{n-1} \cap Q_{n-1}} x_{0}, \quad \forall n \geqslant 1,
\end{array}\right.
$$

where $\theta_{n}=\left(k_{h(n)}^{4}-1\right) \rho_{n}, \rho_{n}=\sup \left\{\left\|x_{n}-p\right\|: p \in \Gamma\right\}<\infty$. When $\left\{\alpha_{n}\right\},\left\{\beta_{n}\right\} \subset(0,1)$ and $\left\{r_{n}\right\}$ satisfying the following conditions:

(i) $\frac{\alpha_{n}}{\beta_{n}} \geqslant s, \forall n \geqslant 0$;

(ii) $\mathrm{r}_{\mathrm{n}} \in[\mathrm{a}, \mathrm{b}]$ for some $0<\mathrm{a}<\mathrm{b}<2 \alpha, \forall \mathrm{n} \geqslant 0$;

(iii) $\liminf _{n \rightarrow \infty} g_{n-1}>0$,

where $g_{n-1}=\left(1-\alpha_{n-1}\right)\left(1-\beta_{n-1}\right)\left[k_{h_{n}}^{2}\left(\beta_{n-1}-s\right)-\left(1-\beta_{n-1}\right)^{2} L^{2} s+\beta_{n-1} s\right], \forall n \geqslant 1$.

Then sequence $\left\{x_{n}\right\}$ converges strongly to $P_{\Gamma} x_{0}$.

Proof. The process of proof is similar to the proof of Theorem 3.4 except $\omega_{w}\left(x_{n}\right) \subseteq \operatorname{GEP}(\Theta, B)$. The proof of $\omega_{w}\left(x_{n}\right) \subseteq \operatorname{GEP}(\Theta, B)$ is similar to the proof of Theorem 3.2, we only replace $x_{n}$ by $u_{n-1}$ and use (3.27). This completes the proof.

Remark 3.6. Similarly, in Theorem 3.4 and Theorem 3.5, if $s_{i}=0,1 \leqslant i \leqslant N$ and the conditions which sequences $\left\{\alpha_{n}\right\}$ and $\left\{\beta_{n}\right\}$ satisfy in Theorem 3.4 and Theorem 3.5 replaced of the following:

(i) $\left\{\alpha_{n}\right\} \subset[0,1),\left\{\beta_{n}\right\} \subset(0,1)$;

(ii) $\limsup _{n \rightarrow \infty} \alpha_{n}<1,0<\liminf _{n \rightarrow \infty} \beta_{n} \leqslant \limsup _{n \rightarrow \infty} \beta_{n}<1$.

Then, two kinds of strong convergence theorems for a finite family of asymptotically nonexpansive mappings can be obtained.

\section{Applications}

In this section, we introduce the several applications of weak convergence theorem (Theorem 3.1). According to different situations, the corresponding strong convergence theorem (Theorem 3.4) has different results.

\subsection{Application to (mixed) equilibrium problem (EP and MEP)}

In (1.2), if $B=\varphi \equiv 0$, the generalized mixed equilibrium problem reduces the equilibrium problem $(\mathrm{EP})$, that is, to find $x \in \mathrm{C}$ such that

$$
\Theta(x, y) \geqslant 0, \quad \forall y \in C .
$$

If $B \equiv 0$, the generalized mixed equilibrium problem reduces the mixed equilibrium problem (MEP), that is, to find $x \in \mathrm{C}$ such that

$$
\Theta(x, y)+\varphi(y)-\varphi(x) \geqslant 0, \quad \forall y \in C .
$$


Therefore, the result of Theorem 3.1 can be applied to (mixed) equilibrium problem (EP and MEP), then, we have the following results.

Theorem 4.1. Let $\mathrm{C}$ be a nonempty closed convex subset of an infinite dimensional real Hilbert space $\mathrm{H}$ and let $\left\{\mathrm{T}_{i}\right\}_{1 \leqslant i \leqslant N}$ be a finite family of $\left\{\mathrm{s}_{i}\right\}_{1 \leqslant i \leqslant N}$-strictly asymptotically pseudo-contractive mappings with sequence $\left\{\mathrm{s}_{i}\right\} \subseteq$ $[0,1)$ and $\left\{k_{n, i}\right\} \subseteq[1, \infty)$ such that $\lim _{n \rightarrow \infty} k_{n, i}=1$ and $\sum_{n=0}^{\infty}\left(k_{n, i}-1\right)<\infty$. Assume that $\Theta: C \times C \rightarrow R$ satisfies (A1)-(A4), $s=\max \left\{s_{i}: 1 \leqslant i \leqslant N\right\},\left\{k_{n}\right\}=\max \left\{k_{n, i}: 1 \leqslant i \leqslant N\right\}$ and $\Gamma=\bigcap_{i=1}^{N} F\left(T_{i}\right) \bigcap E P(\Theta) \neq \emptyset$. Let $\left\{x_{n}\right\}$ be a sequence generated by the following algorithm:

$$
\left\{\begin{array}{l}
x_{0} \in C \text { chosen arbitrarily, } \\
y_{n-1}=\beta_{n-1} x_{n-1}+\left(1-\beta_{n-1}\right) T_{i(n)}^{h(n)} x_{n-1} \\
z_{n-1}=\alpha_{n-1} x_{n-1}+\left(1-\alpha_{n-1}\right) T_{i(n)}^{h(n)} y_{n-1}, \\
x_{n} \in C, \text { such that } \Theta\left(x_{n}, y\right)+\frac{1}{r_{n-1}}\left\langle y-x_{n}, x_{n}-z_{n-1}\right\rangle \geqslant 0, \quad \forall y \in C, \quad n \geqslant 1,
\end{array}\right.
$$

where $\left\{\alpha_{n}\right\},\left\{\beta_{n}\right\} \subset(0,1)$ and $\left\{r_{n}\right\}$ satisfying the following conditions:

(i) $\frac{\alpha_{n}}{\beta_{n}} \geqslant s, \forall n \geqslant 0$;

(ii) $\liminf _{n \rightarrow \infty} r_{n}>0, \forall n \geqslant 0$;

(iii) $\liminf _{\mathrm{n} \rightarrow \infty} \mathrm{g}_{\mathrm{n}-1}>0, \forall \mathrm{n} \geqslant 1$,

where $g_{n-1}=\left(1-\alpha_{n-1}\right)\left(1-\beta_{n-1}\right)\left[k_{h(n)}^{2}\left(\beta_{n-1}-s\right)-\left(1-\beta_{n-1}\right)^{2} L^{2} s+\beta_{n-1} s\right]$.

Then sequence $\left\{x_{n}\right\}$ converges weakly to a point in $\Gamma$.

Theorem 4.2. Let $\mathrm{C}$ be a nonempty closed convex subset of an infinite dimensional real Hilbert space $\mathrm{H}$ and let $\left\{\mathrm{T}_{i}\right\}_{1 \leqslant i \leqslant N}$ be a finite family of $\left\{s_{i}\right\}_{1 \leqslant i \leqslant N}$-strictly asymptotically pseudo-contractive mappings with sequence $\left\{s_{i}\right\} \subseteq[0,1)$ and $\left\{k_{n, i}\right\} \subseteq[1, \infty)$ such that $\lim _{n \rightarrow \infty} k_{n, i}=1$ and $\sum_{n=0}^{\infty}\left(k_{n, i}-1\right)<\infty$. Let $\varphi: C \rightarrow R$ be a proper lower semi-continuous and convex functional. Assume that $\Theta: C \times C \rightarrow R$ satisfies (A1)-(A4), $s=\max \left\{s_{i}: 1 \leqslant i \leqslant N\right\},\left\{k_{n}\right\}=\max \left\{k_{n, i}: 1 \leqslant i \leqslant N\right\}$ and $\Gamma=\bigcap_{i=1}^{N} F\left(T_{i}\right) \bigcap \operatorname{MEP}(\Theta, \varphi) \neq \emptyset$. Let $\left\{x_{n}\right\}$ be a sequence generated by the following algorithm:

$$
\left\{\begin{array}{l}
x_{0} \in C \text { chosen arbitrarily, } \\
y_{n-1}=\beta_{n-1} x_{n-1}+\left(1-\beta_{n-1}\right) T_{i(n)}^{h(n)} x_{n-1}, \\
z_{n-1}=\alpha_{n-1} x_{n-1}+\left(1-\alpha_{n-1}\right) T_{i(n)}^{h(n)} y_{n-1}, \\
x_{n} \in C, \text { such that } \Theta\left(x_{n}, y\right)+\varphi(y)-\varphi\left(x_{n}\right)+\frac{1}{r_{n-1}}\left\langle y-x_{n}, x_{n}-z_{n-1}\right\rangle \geqslant 0, \quad \forall y \in C, \quad n \geqslant 1,
\end{array}\right.
$$

where $\left\{\alpha_{n}\right\},\left\{\beta_{n}\right\} \subset(0,1)$ and $\left\{r_{n}\right\}$ satisfying the following conditions:

(i) $\frac{\alpha_{n}}{\beta_{n}} \geqslant s, \forall n \geqslant 0$;

(ii) $\liminf _{n \rightarrow \infty} r_{n}>0, \forall n \geqslant 0$;

(iii) $\liminf _{\mathrm{n} \rightarrow \infty} \mathrm{g}_{\mathrm{n}-1}>0, \forall \mathrm{n} \geqslant 1$,

where $g_{n-1}=\left(1-\alpha_{n-1}\right)\left(1-\beta_{n-1}\right)\left[k_{h(n)}^{2}\left(\beta_{n-1}-s\right)-\left(1-\beta_{n-1}\right)^{2} L^{2} s+\beta_{n-1} s\right]$.

Then sequence $\left\{x_{n}\right\}$ converges weakly to a point in $\Gamma$.

\subsection{Application to (mixed) variational inequality}

A variational inequality problem (VIP) is to find $x \in \mathrm{C}$ such that

$$
\langle\mathrm{B} x, y-x\rangle \geqslant 0, \quad \forall y \in C .
$$

The solution set of VIP is denoted by $\mathrm{VI}(\mathrm{B}, \mathrm{C})$. 
The mixed variational inequality is to find $x \in C$ such that

$$
\langle\mathrm{B} x, y-x\rangle+\varphi(y)-\varphi(x) \geqslant 0, \quad \forall y \in C .
$$

We denote the solution set of (4.1) with $\mathrm{VI}(\mathrm{B}, \mathrm{C}, \varphi)$.

If $\Theta=\varphi \equiv 0$, the generalized mixed equilibrium problem reduces a variational inequality problem.

If $\Theta \equiv 0$, the generalized mixed equilibrium problem reduces the mixed variational inequality.

Putting $F(x, y)=\langle B x, y-x\rangle$, if $B$ is an $\alpha$-inverse-strongly monotone mapping, we can easily show that $\mathrm{F}$ satisfies conditions (A1)-(A4). Then, the following theorems can be obtained from Theorem 3.1.

Theorem 4.3. Let $\mathrm{C}$ be a nonempty closed convex subset of an infinite dimensional real Hilbert space $\mathrm{H}$ and let $\left\{\mathrm{T}_{i}\right\}_{1 \leqslant i \leqslant N}$ be a finite family of $\left\{\mathrm{s}_{i}\right\}_{1 \leqslant i \leqslant N}$-strictly asymptotically pseudo-contractive mappings with sequence $\left\{s_{i}\right\} \subseteq[0,1)$ and $\left\{k_{n, i}\right\} \subseteq[1, \infty)$ such that $\lim _{n \rightarrow \infty} k_{n, i}=1$ and $\sum_{n=0}^{\infty}\left(k_{n, i}-1\right)<\infty$. Let $\mathrm{B}: \mathrm{C} \rightarrow \mathrm{H}$ be an $\alpha$-inverse-strongly monotone mapping. Assume that $s=\max \left\{s_{i}: 1 \leqslant i \leqslant N\right\},\left\{k_{n}\right\}=\max \left\{k_{n, i}: 1 \leqslant i \leqslant N\right\}$ and $\Gamma=\bigcap_{i=1}^{N} \mathrm{~F}\left(\mathrm{~T}_{i}\right) \bigcap \mathrm{VI}(\mathrm{B}, \mathrm{C}) \neq \emptyset$. Let $\left\{x_{\mathrm{n}}\right\}$ be a sequence generated by the following algorithm:

$$
\left\{\begin{array}{l}
x_{0} \in C \text { chosen arbitrarily, } \\
y_{n-1}=\beta_{n-1} x_{n-1}+\left(1-\beta_{n-1}\right) T_{i(n)}^{h(n)} x_{n-1}, \\
z_{n-1}=\alpha_{n-1} x_{n-1}+\left(1-\alpha_{n-1}\right) T_{i(n)}^{h(n)} y_{n-1} \\
x_{n} \in C, \quad \text { such that }\left\langle B x_{n}, y-x_{n}\right\rangle+\frac{1}{r_{n-1}}\left\langle y-x_{n}, x_{n}-z_{n-1}\right\rangle \geqslant 0, \quad \forall y \in C, n \geqslant 1,
\end{array}\right.
$$

where $\left\{\alpha_{n}\right\},\left\{\beta_{n}\right\} \subset(0,1)$ and $\left\{r_{n}\right\}$ satisfying the following conditions:

(i) $\frac{\alpha_{n}}{\beta_{n}} \geqslant s, \forall n \geqslant 0$;

(ii) $\liminf _{n \rightarrow \infty} r_{n}>0, \forall n \geqslant 0$;

(iii) $\liminf _{\mathrm{n} \rightarrow \infty} \mathrm{g}_{\mathrm{n}-1}>0, \forall \mathrm{n} \geqslant 1$,

where $g_{n-1}=\left(1-\alpha_{n-1}\right)\left(1-\beta_{n-1}\right)\left[k_{h(n)}^{2}\left(\beta_{n-1}-s\right)-\left(1-\beta_{n-1}\right)^{2} L^{2} s+\beta_{n-1} s\right]$.

Then sequence $\left\{x_{n}\right\}$ converges weakly to a point in $\Gamma$.

Theorem 4.4. Let $\mathrm{C}$ be a nonempty closed convex subset of an infinite dimensional real Hilbert space $\mathrm{H}$ and let $\left\{\mathrm{T}_{i}\right\}_{1 \leqslant i \leqslant N}$ be a finite family of $\left\{\mathrm{s}_{i}\right\}_{1 \leqslant i \leqslant N}$-strictly asymptotically pseudo-contractive mappings with sequence $\left\{s_{i}\right\} \subseteq[0,1)$ and $\left\{k_{n, i}\right\} \subseteq[1, \infty)$ such that $\lim _{n \rightarrow \infty} k_{n, i}=1$ and $\sum_{n=0}^{\infty}\left(k_{n, i}-1\right)<\infty$. Let $\varphi: C \rightarrow R$ be a proper lower semi-continuous and convex functional and $\mathrm{B}: \mathrm{C} \rightarrow \mathrm{H}$ be an $\alpha$-inverse-strongly monotone mapping. Assume that $s=\max \left\{s_{i}: 1 \leqslant i \leqslant N\right\},\left\{k_{n}\right\}=\max \left\{k_{n, i}: 1 \leqslant i \leqslant N\right\}$ and $\Gamma=\bigcap_{i=1}^{N} \mathrm{~F}\left(\mathrm{~T}_{i}\right) \bigcap \mathrm{VI}(\mathrm{B}, \mathrm{C}, \varphi) \neq \emptyset$. Let $\left\{x_{n}\right\}$ be a sequence generated by the following algorithm:

$$
\left\{\begin{array}{l}
x_{0} \in C \text { chosen arbitrarily, } \\
y_{n-1}=\beta_{n-1} x_{n-1}+\left(1-\beta_{n-1}\right) T_{i(n)}^{h(n)} x_{n-1}, \\
z_{n-1}=\alpha_{n-1} x_{n-1}+\left(1-\alpha_{n-1}\right) T_{i(n)}^{h(n)} y_{n-1}, \\
x_{n} \in C, \quad \text { such that }\left\langle B x_{n}, y-x_{n}\right\rangle+\varphi(y)-\varphi\left(x_{n}\right)+\frac{1}{r_{n-1}}\left\langle y-x_{n}, x_{n}-z_{n-1}\right\rangle \geqslant 0, \quad \forall y \in C, n \geqslant 1,
\end{array}\right.
$$

where $\left\{\alpha_{n}\right\},\left\{\beta_{n}\right\} \subset(0,1)$ and $\left\{r_{n}\right\}$ satisfying the following conditions:

(i) $\frac{\alpha_{n}}{\beta_{n}} \geqslant s, \forall n \geqslant 0$;

(ii) $\liminf _{n \rightarrow \infty} r_{n}>0, \forall n \geqslant 0$;

(iii) $\liminf _{n \rightarrow \infty} g_{n-1}>0, \forall n \geqslant 1$, where $g_{n-1}=\left(1-\alpha_{n-1}\right)\left(1-\beta_{n-1}\right)\left[k_{h(n)}^{2}\left(\beta_{n-1}-s\right)-\left(1-\beta_{n-1}\right)^{2} L^{2} s+\beta_{n-1} s\right]$.

Then sequence $\left\{x_{n}\right\}$ converges weakly to a point in $\Gamma$. 


\subsection{Application to convex minimization problem}

In (1.2), if $\Theta=B \equiv 0$, the generalized mixed equilibrium problem reduces a convex minimization problem, that is, to find $x \in C$ such that

$$
\varphi(y) \geqslant \varphi(x), \quad \forall y \in C .
$$

We denote the solution set of (4.2) with $\operatorname{CMP}(\varphi)$.

Therefore, Theorem 3.1 can reduce the following theorem about convex minimization problem.

Theorem 4.5. Let $\mathrm{C}$ be a nonempty closed convex subset of an infinite dimensional real Hilbert space $\mathrm{H}$ and let $\left\{T_{i}\right\}_{1 \leqslant i \leqslant N}$ be a finite family of $\left\{s_{i}\right\}_{1 \leqslant i \leqslant N}$-strictly asymptotically pseudo-contractive mappings with sequence $\left\{s_{i}\right\} \subseteq$ $[0,1)$ and $\left\{k_{n, i}\right\} \subseteq[1, \infty)$ such that $\lim _{n \rightarrow \infty} k_{n, i}=1$ and $\sum_{n=0}^{\infty}\left(k_{n, i}-1\right)<\infty$. Let $\varphi: C \rightarrow R$ be a proper lower semi-continuous and convex functional. Assume that $s=\max \left\{s_{i}: 1 \leqslant i \leqslant N\right\},\left\{k_{n}\right\}=\max \left\{k_{n, i}: 1 \leqslant i \leqslant N\right\}$ and $\Gamma=\bigcap_{i=1}^{N} \mathrm{~F}\left(\mathrm{~T}_{i}\right) \bigcap \operatorname{CMP}(\varphi) \neq \emptyset$. Let $\left\{x_{n}\right\}$ be a sequence generated by the following algorithm:

$$
\left\{\begin{array}{l}
x_{0} \in C \text { chosen arbitrarily, } \\
y_{n-1}=\beta_{n-1} x_{n-1}+\left(1-\beta_{n-1}\right) T_{i(n)}^{h(n)} x_{n-1}, \\
z_{n-1}=\alpha_{n-1} x_{n-1}+\left(1-\alpha_{n-1}\right) T_{i(n)}^{h(n)} y_{n-1}, \\
x_{n} \in C, \text { such that } \varphi(y)-\varphi\left(x_{n}\right)+\frac{1}{r_{n-1}}\left\langle y-x_{n}, x_{n}-z_{n-1}\right\rangle \geqslant 0, \quad \forall y \in C, n \geqslant 1,
\end{array}\right.
$$

where $\left\{\alpha_{n}\right\},\left\{\beta_{n}\right\} \subset(0,1)$ and $\left\{r_{n}\right\}$ satisfying the following conditions:

(i) $\frac{\alpha_{n}}{\beta_{n}} \geqslant s, \forall n \geqslant 0$;

(ii) $\liminf _{n \rightarrow \infty} r_{n}>0, \forall n \geqslant 0$;

(iii) $\liminf _{n \rightarrow \infty} g_{n-1}>0, \forall n \geqslant 1$,

where $g_{n-1}=\left(1-\alpha_{n-1}\right)\left(1-\beta_{n-1}\right)\left[k_{h(n)}^{2}\left(\beta_{n-1}-s\right)-\left(1-\beta_{n-1}\right)^{2} L^{2} s+\beta_{n-1} s\right]$.

Then sequence $\left\{x_{n}\right\}$ converges weakly to a point in $\Gamma$.

4.4. Application to convex feasibility problem

The convex feasibility problem for a family of mappings $\left\{T_{i}\right\}_{1 \leqslant i \leqslant N}$ is to find a point $x$ such that $x \in \bigcap_{i=1}^{N} F\left(T_{i}\right)$.

Therefore, Theorem 3.1 can also reduce the following theorem about convex feasibility problem.

Theorem 4.6. Let $\mathrm{C}$ be a nonempty closed convex subset of an infinite dimensional real Hilbert space $\mathrm{H}$ and let $\left\{\mathrm{T}_{i}\right\}_{1 \leqslant i \leqslant N}$ be a finite family of $\left\{\mathrm{s}_{i}\right\}_{1 \leqslant i \leqslant N}$-strictly asymptotically pseudo-contractive mappings with sequence $\left\{s_{i}\right\} \subseteq[0,1)$ and $\left\{k_{n, i}\right\} \subseteq[1, \infty)$ such that $\lim _{n \rightarrow \infty} k_{n, i}=1$ and $\sum_{n=0}^{\infty}\left(k_{n, i}-1\right)<\infty$. Assume that $s=$ $\max \left\{s_{i}: 1 \leqslant i \leqslant N\right\},\left\{k_{n}\right\}=\max \left\{k_{n, i}: 1 \leqslant i \leqslant N\right\}$ and $\Gamma=\bigcap_{i=1}^{N} F\left(T_{i}\right) \neq \emptyset$. Let $\left\{x_{n}\right\}$ be a sequence generated by the following algorithm:

$$
\left\{\begin{array}{l}
x_{0} \in C \text { chosen arbitrarily, } \\
y_{n-1}=\beta_{n-1} x_{n-1}+\left(1-\beta_{n-1}\right) T_{i(n)}^{h(n)} x_{n-1} \\
z_{n-1}=\alpha_{n-1} x_{n-1}+\left(1-\alpha_{n-1}\right) T_{i(n)}^{h(n)} y_{n-1} \\
x_{n}=P_{C} z_{n-1}
\end{array}\right.
$$

where $\left\{\alpha_{n}\right\},\left\{\beta_{n}\right\} \subset(0,1)$ and $\left\{r_{n}\right\}$ satisfying the following conditions:

(i) $\frac{\alpha_{n}}{\beta_{n}} \geqslant s, \forall n \geqslant 0$;

(ii) $\liminf _{n \rightarrow \infty} r_{n}>0, \forall n \geqslant 0$;

(iii) $\liminf _{\mathrm{n} \rightarrow \infty} \mathrm{g}_{\mathrm{n}-1}>0, \forall \mathrm{n} \geqslant 1$,

where $g_{n-1}=\left(1-\alpha_{n-1}\right)\left(1-\beta_{n-1}\right)\left[k_{h(n)}^{2}\left(\beta_{n-1}-s\right)-\left(1-\beta_{n-1}\right)^{2} L^{2} s+\beta_{n-1} s\right]$.

Then sequence $\left\{x_{n}\right\}$ converges weakly to a point in $\Gamma$. 


\section{Numerical experiments}

In this section, respectively, we give the corresponding numerical examples of Theorem 3.1 and Theorem 3.2.

Example 5.1. Let $H:=R$ and $C:=[-1,1] \subset R$. For all $x, y \in C, \Theta(x, y)=y^{2}+x y-2 x^{2}, B x=2 x$, $\varphi(x)=\frac{1}{2} x^{2}$. It is obvious that $\Theta: C \times C \rightarrow R$ is a real-valued bifunction satisfying the following conditions (A1)-(A4), B : C $\rightarrow \mathrm{H}$ is a monotone mapping and $\varphi: \mathrm{C} \rightarrow \mathrm{R}$ is a continuous and convex functional.

Then, for given $r>0$ and $x \in H$, by Lemma 2.1, there exists a unique $u \in C$ such that

$$
\begin{gathered}
\Theta(u, y)+\langle B u, y-u\rangle+\varphi(y)-\varphi(u)+\frac{1}{r}\langle y-u, u-x\rangle \geqslant 0, \quad \forall y \in C . \\
\mathbb{1} \\
y^{2}+u y-2 u^{2}+2 u(y-u)+\frac{1}{2} y^{2}-\frac{1}{2} u^{2}+\frac{1}{r}(y-u)(u-x) \geqslant 0, \quad \forall y \in C . \\
\mathbb{1} \\
3 r y^{2}+(6 r u+2 u-2 x) y+2 u x-2 u-9 r u^{2} \geqslant 0, \quad \forall y \in C .
\end{gathered}
$$

Let $F(y)=3 r y^{2}+(6 r u+2 u-2 x) y+2 u x-2 u-9 r u^{2}$. Then discriminant $\Delta$ of $F(y)$ is

$$
\Delta=(6 r u+2 u-2 x)^{2}-4 \times 3 r y\left(2 u x-2 u-9 r u^{2}\right)=[2 x-2 u(1+6 r)]^{2} .
$$

Taking $\Delta \leqslant 0$, then for all $y \in C, F(y) \geqslant 0$. Again from uniqueness of $u$, we have that

$$
\mathrm{u}=\mathrm{K}_{\mathrm{r}} \mathrm{x}=\frac{1}{1+6 \mathrm{r}} \mathrm{x} .
$$

From algorithm (3.1), we can obtain that

$$
\left\{\begin{aligned}
y_{n-1} & =\beta_{n-1} x_{n-1}+\left(1-\beta_{n-1}\right) T_{i(n)}^{h(n)} x_{n-1}, \\
z_{n-1} & =\alpha_{n-1} x_{n-1}+\left(1-\alpha_{n-1}\right) T_{i(n)}^{h(n)} y_{n-1}, \\
x_{n} & =\frac{1}{1+6 r_{n-1}} z_{n-1} .
\end{aligned}\right.
$$

Let $N=2, T_{1} x=x$, for all $x \in[-1,1]$,

$$
\mathrm{T}_{2} x:=\left\{\begin{array}{c}
x, \quad x \in[-1,0) \\
\frac{1}{2} x, \quad x \in[0,1]
\end{array}\right.
$$

and taking $x_{0}=1, \alpha_{n}=\frac{1}{n+1}, \beta_{n}=\frac{n}{2(n+1)}, r_{n}=\frac{n}{4(n+1)}$. We have the following numerical results:

Table 1: numerical examples of Theorem 3.1.

\begin{tabular}{ccccccccc}
\hline $\mathrm{n}$ & 0 & 1 & 2 & 3 & 4 & 5 & 6 & $\ldots$ \\
$x_{\mathrm{n}}$ & 1 & 1 & 0.3750 & 0.1875 & 0.0308 & 0.0140 & 0.0014 & $\ldots$ \\
\hline
\end{tabular}




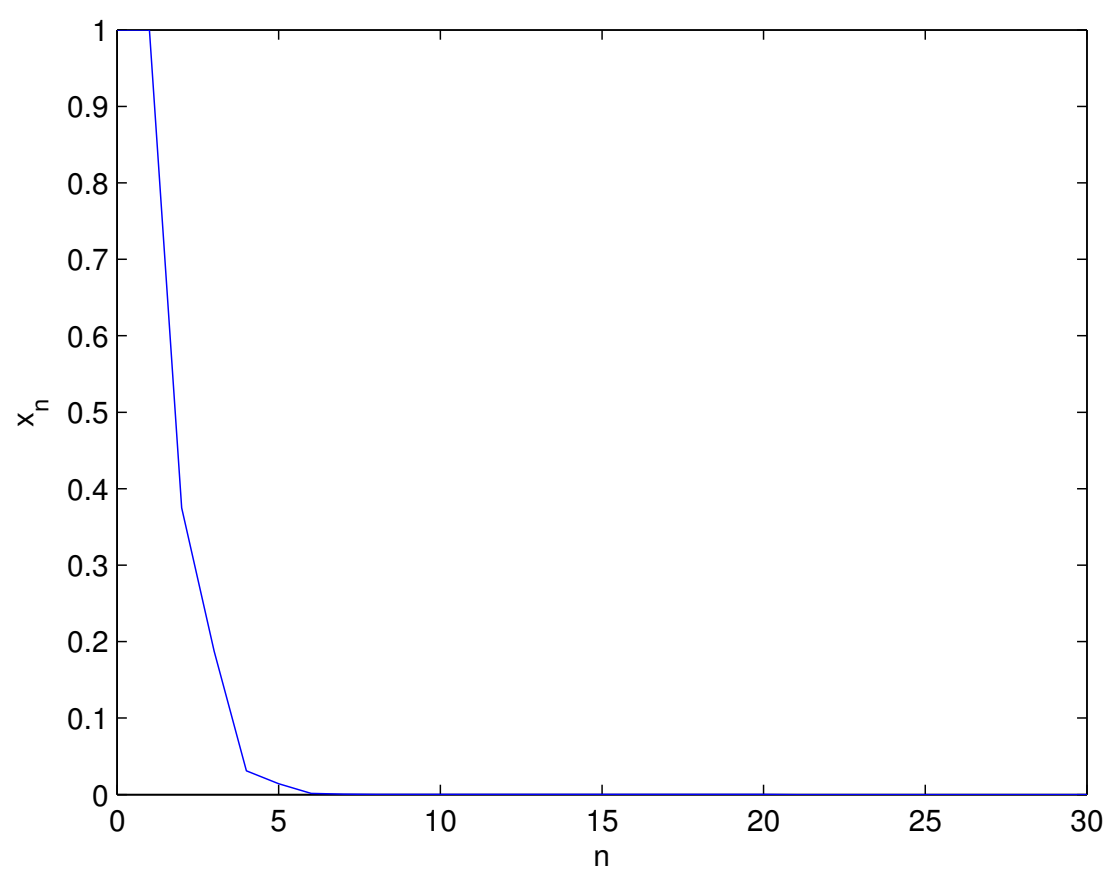

Figure 1: numerical examples of Theorem 3.1.

Example 5.2. Let $H:=R$ and $C:=[-1,1] \subset R$. For all $x, y \in C, \Theta(x, y)=y^{2}+x y-2 x^{2}, B x=2 x$. Then, for given $r>0$ and $x \in H$, by Lemma 2.1, there exists a unique $u \in C$ such that

$$
\Theta(u, y)+\frac{1}{r}\langle y-u, u-x\rangle \geqslant 0, \quad \forall y \in C
$$

Similar to the above method, we can obtain that

$$
u=T_{r} x=\frac{1}{1+3 r}
$$

From algorithm (3.20), we can obtain that

$$
\left\{\begin{aligned}
y_{n-1} & =\beta_{n-1} x_{n-1}+\left(1-\beta_{n-1}\right) T_{i(n)}^{h(n)} x_{n-1}, \\
z_{n-1} & =\alpha_{n-1} x_{n-1}+\left(1-\alpha_{n-1}\right) T_{i(n)}^{h(n)} y_{n-1}, \\
x_{n} & =T_{r_{n-1}}\left(z_{n-1}-r_{n-1} B z_{n-1}\right)=\frac{1-2 r_{n-1}}{1+3 r_{n-1}} z_{n-1} .
\end{aligned}\right.
$$

Let $N, T_{1}, T_{2}, \alpha_{n}, \beta_{n}, r_{n}, x_{0}$ are the same as before.

Table 2: numerical examples of Theorem 3.2.

\begin{tabular}{ccccccccc}
\hline $\mathrm{n}$ & 0 & 1 & 2 & 3 & 4 & 5 & 6 & $\ldots$ \\
$\mathrm{x}_{\mathrm{n}}$ & 1 & 1 & 0.3580 & 0.1591 & 0.0222 & 0.0083 & $6.5189 \times 10^{-4}$ & $\ldots$ \\
\hline
\end{tabular}




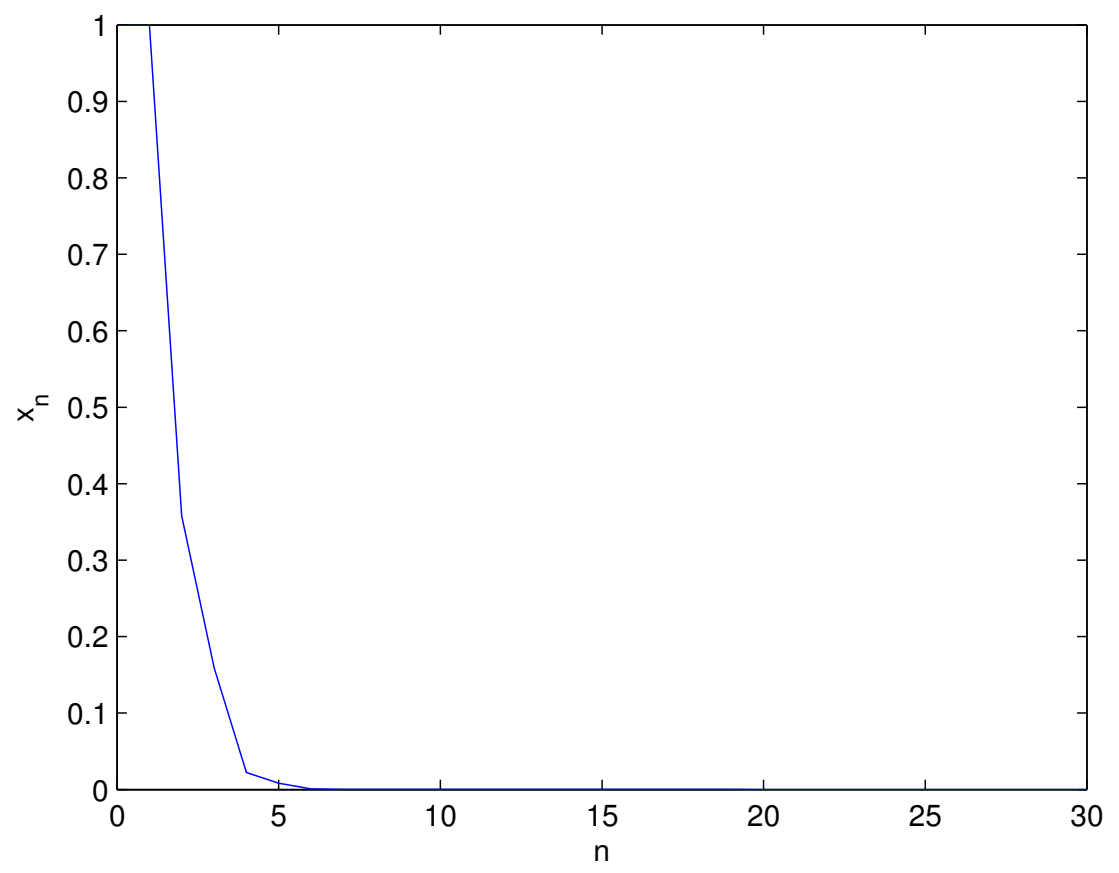

Figure 2: numerical examples of Theorem 3.2.

\section{Acknowledgment}

The authors are most grateful to the referees and the editor for their helpful comments and advices which help to improve the contents of this paper.

\section{References}

[1] G. L. Acedo, H.-K. Xu, Iterative methods for strict pseudo-contractions in Hilbert spaces, Nonlinear Anal., 67 (2007), 2258-2271. 2.5

[2] B. A. Bin Dehaish, A. Latif, H. O. Bakodah, X.-L. Qin, A regularization projection algorithm for various problems with nonlinear mappings in Hilbert spaces, J. Inequal. Appl., 2015 (2015), 14 pages. 1

[3] B. A. Bin Dehaish, X.-L. Qin, A. Latif, H. O. Bakodah, Weak and strong convergence of algorithms for the sum of two accretive operators with applications, J. Nonlinear Convex Anal., 16 (2015), 1321-1336. 2

[4] E. Blum, W. Oettli, From optimization and variational inequalities to equilibrium problems, Math. Student, 63 (1994), 123-145. 2.1

[5] S.-S. Chang, H. B. Thompson, G. X.-Z. Yuan, The existence theorems of solutions for generalized vector-valued variationallike inequalities, Comput. Math. Appl., 37 (1999), 1-9. 1

[6] S. Y. Cho, B. A. Bin Dehaish, X.-L. Qin, Weak convergence of a splitting algorithm in Hilbert spaces, J. Appl. Anal. Comput., 7 (2017), 427-438. 1

[7] S. Y. Cho, S. M. Kang, Approximation of fixed points of pseudocontraction semigroups based on a viscosity iterative process, Appl. Math. Lett., 24 (2011), 224-228. 1

[8] S. Y. Cho, X.-L. Qin, L. Wang, Strong convergence of a splitting algorithm for treating monotone operators, Fixed Point Theory Appl., 2014 (2014), 15 pages. 2

[9] N.-N. Fang, Some results on split variational inclusion and fixed point problems in Hilbert spaces, Commun. Optim. Theory, 2017 (2017), 14 pages. 1

[10] J. S. Jung, Weak convergence theorems for generalized mixed equilibrium problems, monotone mappings and pseudocontractive mappings, J. Korean Math. Soc., 52 (2015), 1179-1194. 2.1, 2

[11] J. K. Kim, S. Y. Cho, X.-L. Qin, Some results on generalized equilibrium problems involving strictly pseudocontractive mappings, Acta Math. Sci. Ser. B Engl. Ed., 31 (2011), 2041-2057. 1, 2.4

[12] J. K. Kim, Y. M. Nam, J. Y. Sim, Convergence theorems of implicit iterative sequences for a finite family of asymptotically quasi-nonxpansive type mappings, Nonlinear Anal., 71 (2009), e2839-e2848. 1 
[13] Y. Liu, Convergence theorems for generalized equilibrium problems and asymptotically k-strict pseudo-contractions in Hilbert spaces, East Asian Math. J., 29 (2013), 303-314. 1, 1

[14] C. Martinez-Yanes, H.-K. Xu, Strong convergence of the CQ method for fixed point iteration processes, Nonlinear Anal., 64 (2006), 2400-2411. 2.6

[15] X.-L. Qin, S. Y. Cho, Convergence analysis of a monotone projection algorithm in reflexive banach spaces, Acta Math. Sci. Ser. B Engl. Ed., 37 (2017), 488-502. 1

[16] X.-L. Qin, Y. J. Cho, S. M. Kang, M.-J. Shang, A hybrid iterative scheme for asymptotically k-strict pseudo-contractions in Hilbert spaces, Nonlinear Anal., 70 (2009), 1902-1911. 1, 2.3

[17] X.-L. Qin, S. Y. Cho, L. Wang, A regularization method for treating zero points of the sum of two monotone operators, Fixed Point Theory Appl., 2014 (2014), 10 pages. 2

[18] G. S. Saluja, Weak convergence theorems for nearly asymptotically nonexpansive mappings and asymptotically nonexpansive non-self mappings in uniformly convex Banach spaces, Commun. Optim. Theory, 2017 (2017), 23 pages. 1

[19] Y.-F. Su, M.-J. Shang, X.-L. Qin, An iterative method of solution for equilibrium and optimization problems, Nonlinear Anal., 69 (2008), 2709-2719. 1

[20] K.-K. Tan, H.-K. Xu, Approximating fixed points of nonexpansive mappings by the Ishikawa iteration process, J. Math. Anal. Appl., 178 (1993), 301-308. 2.2

[21] I. Yildirim, Approximating fixed points of two finite families of I-asymptotically quasi-nonexpansive mappings, J. Nonlinear Funct. Anal., 2016 (2016), 17 pages. 1

[22] M.-L. Zhang, An algorithm for treating asymptotically strict pseudocontractions and monotone operators, Fixed Point Theory Appl., 2014 (2014), 14 pages. 1

[23] M.-L. Zhang, Strong convergence of a viscosity iterative algorithm in Hilbert spaces, J. Nonlinear Funct. Anal., 2014 (2014), 16 pages. 1

[24] Q.-N. Zhang, Nonlinear operators, equilibrium problems and monotone projection algorithms, Commun. Optim. Theory, 2017 (2017), 11 pages. 1 